\title{
The meter of Tashlhiyt Berber songs
}

\author{
Tomas Riad 1
}

Received: 12 April 2014 / Accepted: 16 August 2016

(C) The Author(s) 2016. This article is published with open access at Springerlink.com

\begin{abstract}
I present an analysis of Tashlhiyt Berber meter, based on the corpus of 'straight' meters collected and analyzed by Dell and Elmedlaoui (2008). There are 56 meters (35 independent, 21 dependent), which are systematically related to each other. Based on the many shared properties of all straight meters and their individual inflexibility, I suggest that they all derive from the same general metrical template, much in the same way as proposed by Deo (2007) for several Sanskrit meters. The individual meters are thus different realizations of the general meter, where the modulation of line length is part of the realizational variation (unlike the case in Sanskrit). I also argue that the generalizations pertaining to the system of meters can be better understood, and more broadly formulated, in terms of the phonology of the language, in three areas. First, the Tashlhiyt straight meters are rhythmic in a very specific sense: they all obey NoCLASH. Several previously unconnected facts-verse foot shapes, avoidance of two heavy syllables in sequence, and the regular alternation of verse feet-follow from this single fact. Second, the meters are pervasively binary, with a strong preference for tetrameter, a fact that is not predicted by models where line length is stipulated (e.g. Hanson and Kiparsky 1996; Fabb and Halle 2008). Line length tendencies follow from the linguistic constraints on binarity, under the assumption that metrical templates are derived from the prosodic hierarchy, rather than externally, e.g. from a specific meter-generating module (e.g. Kiparsky 1977; Blumenfeld 2015). This would indicate that meter is derived from grammar in just the same way as prosodic morphemes. Third, and related to the second point, the regular prosody and the meter are simultaneously present in a line of verse, a common assumption. However, the fact that both structures come out of the linguistic grammar means that grammar operates in both domains (like in root-and-pattern morphology), rather than there being a matching between them. Meter obeys some constraint to a
\end{abstract}

T. Riad

tomas.riad@su.se

1 Department of Swedish Language and Multilingualism, Stockholm University, 10691 Stockholm, Sweden 
higher degree than does regular prosody, instantiating overall improvement in the particular respect addressed by the chosen constraint. In the straight meters of Tashlhiyt Berber, this privileged constraint is NoCLASH (as also in Tegnér's Swedish hexameter), whereas in other systems the constraint may concern e.g. the alignment of prominence at some level (Darío's Spanish alexandrines, Strindberg's Swedish hexameter).

Keywords Poetic meter · Verse feet · Catalexis · Clash · Lapse $\cdot$ Prosodic morphology $\cdot$ Tashlhiyt Berber $\cdot$ Meter and language

\section{Introduction}

Tashlhiyt Berber songs derive from a tradition of western Morocco. As analyzed by Jouad (1995) and Dell and Elmedlaoui (2008), the text of the songs is metered poetry. The meter is quantitative like Greek, Arabic and Japanese. The work of Jouad (1993, 1995) establishes the central fact that the number of syllables per line is strict for any given meter, and that the distribution of syllable quantity, hence light and heavy syllables, is tightly regulated. The work of Dell and Elmedlaoui (2008) adds a number of observations and provides a coherent and inclusive analysis of a large natural class of meters, constituting about half of the meters that they collected in their fieldwork. They call this class the 'straight' meters.

In this article, I look at the same set of meters from the perspective of prosodic metrics (Golston and Riad 2000, 2005), where the general program is to bring metrics under the purview of linguistic prosody, as directly and explicitly as possible. The goal is to improve the adequacy for the description of this group of meters as a system, as well as to provide the outline of a theoretical account of how the generalizations for meter come out of the grammar of Berber. The main claim is that the Tashlhiyt straight meters are defined by the workings of a single linguistic constraint, namely NoClash. We will find that this constraint is always met in the meter, and that several of the observations that have been made regarding the empirical patterning follow directly from the strict obedience of NOCLASH.

The decisive influence on a meter by a single linguistic constraint invites a rethinking of the relationship between language and meter. In line with Golston and Riad (2000, 2005), I assume that the regular prosodic tree and the meter are homologous, which means that the meter is built with authentic units of prosody, just like prosodic morphemes (McCarthy and Prince 1986). Indeed, the proposal is that meter is a prosodic morpheme, but deriving from a higher-order category of the prosodic hierarchy, typically the intonation phrase (IPh). Like the type of prosodic morphology known as root-and-pattern, meter is simultaneous with the regular prosody (unlike reduplication and nickname formation).

Given the two simultaneous, linguistic trees we can understand the role of NoCLASH in Tashlhiyt meter in terms of the notion of a privileged constraint which causes overall prosodic improvement in a meter, in the particular respect defined by the linguistic constraint selected by poets. If this is on the right track, at least for some types of meter, then the notion of matching of meter to prosody, common in many 
models of metrics, is not relevant. Instead, the linguistic grammar operates on both regular prosody and meter, though very selectively on meter.

When we look at how the straight meters relate to one another, we find that they circumscribe an internally coherent metrical space, which contains very few accidental gaps. Indeed, the systematic similarity between the straight meters, in combination with the strictness of realization, suggests that there is really just a single meter at the right level of abstraction. Individual meters are instantiations of this abstract meter. Another system that has been shown to contain meters of this kind is Classical Sanskrit (Deo 2007). The proposal here is that the straight meters of Tashlhiyt Berber exhibit the same type of relation between a basic meter and a number of strict surface variations on that meter. Of the three properties that are typical of Sanskrit metersaperiodicity, invariance and a rich repertoire - the last two hold also for Tashlhiyt Berber. Each line of a meter has a unique, invariant shape, and the repertoire is indeed quite rich, constituted by 56 straight meters, all based on one and the same abstract shape. The Berber meters are however also clearly periodic. They all exhibit the same regularities in the alternation of verse feet across the line. Another interesting fact that sets Berber meter apart from the Sanskrit ones is that line length is not specified at the level of the abstract meter. Rather, the modulation of line length in the individual instantiations of the basic meter is the main factor that yields the large number of distinct meters, beside some shape variation within one of the verse foot types. As is often the case with quantitative meters, the notion of rhythm is not straightforward, and we would agree with Dell and Elmedlaoui (2008: 3) that it is not clear that there is rhythmic periodicity in any constitutive sense. In our analysis, the meter is however sensitive to the rhythmic linguistic constraints NoCLASH and NOLAPSE.

In Sect. 2, we shall take a general look at the relationship between language and meter and introduce the notion of 'privileged' constraints. Thereafter we turn to Tashlhiyt Berber meter, where we look first at the generalizations established in previous scholarship (Sect. 3) and then show how these generalizations can be connected to the central role of NoCLASH (Sect. 4). We then take a look at the entire set of meters (Sect. 5) and demonstrate how the meters fill out a coherent metrical space characterized by minimal variation (Sect. 6). In Sect. 7 we return to the relationship between language and meter and discuss how the Tashlhiyt system should be understood, in terms of its privileged constraint as well as regarding how the difference between the meters should be captured. Section 8 concludes the article.

\section{The relationship between meter and grammar}

In order to make sense of the idea that one single constraint (NOCLASH), taken directly from the grammar, accounts for most properties of Berber meter, a number of assumptions must be made explicit regarding the relationship between meter and grammar.

The general idea in this study is that most properties of meter derive directly from the grammar. This means, first of all, that metrical templates are not artefacts, but products of the grammar, in just the same way as so-called prosodic morphemes 
(McCarthy and Prince 1986, 1994) are. A familiar property of typical prosodic morphemes (reduplicants, nicknames, root-and-pattern templates) is that they tend to exhibit unmarkedness, such as canonical syllable structure or binary shape. Unmarkedness is taken to emerge from the ranking of base $\leftrightarrow$ prosodic morpheme faithfulness constraints below markedness constraints referring to prosodic shape or segmental content. I will assume that a meter is the same kind of object as a prosodic morpheme, but constituted of a higher-order category of the prosodic hierarchy, typically an intonation phrase (IPh). More familiar prosodic morphemes are constituted by syllables, prosodic feet or prosodic words. This accounts for the complexity of meter compared to, say, reduplicants, as a simple function of size and level in the hierarchy. An IPh contains several prosodic categories while a prosodic word contains fewer. Importantly, the unmarked binarity of the categories of the prosodic hierarchy can now be predicted to show through as a tendency in meter. This is what we find in Tashlhiyt Berber as well as elsewhere, where the overall relatively more binary meters are also more common (cf. Sect. 6.2). In Classical Greek, anapestic tetrameter is the most flexible and natural, as well as the most binary of the meters. In Swedish, trochaic tetrameter is common and natural to the language, if sometimes considered a little artless.

The metrical tree, then, is an unmarked rendering of the prosodic hierarchy of the language in question (Golston and Riad 2000). Any deviation from binarity will be marked, hence an underlying property (discussed in Sect. 7.3). This means that the various categories of the metrical tree will be the same as those of the regular prosodic tree of the language at hand. Thus, it is the question of homology rather than resemblance (Sect. 7).

Both trees are co-present in the line of text, and we will argue that such copresence also holds with root-and-pattern morphology, which is thereby the type of prosodic morphology that is the most similar to meter. The parallel trees are illustrated in (1), where the same labels (IPh, PrPh, PrWd) are used above the line of text (=regular prosody) as well as below (=meter). Some traditional terminology is added to the metrical tree for clarity.

\section{Simultaneous trees (sentence from Selkirk 2000)}

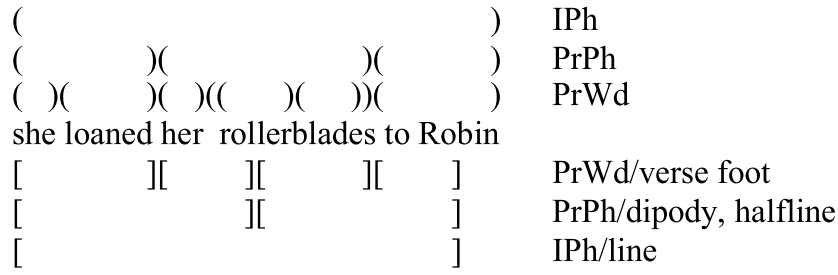

Tradition refers to the categories of meter with a separate terminology, e.g. 'metrical position', 'verse foot', 'metron', 'dipody', 'colon', 'halfline', 'line', 'couplet'. Some of these (depending on how they are defined in each system) will in fact just be alternative names for prosodic categories that are manifest in the language. Thus, the metrical position is usually a prosodic foot or a syllable, the verse foot is typically a 
prosodic word, the dipody or the halfline is often a prosodic phrase and the line of meter is often an intonation phrase, sometimes two intonation phrases. ${ }^{1}$

The meter does not respect word breaks (rol][ler-) and it ignores the last syllable of Robin, which is thereby extrametrical (from the perspective of the meter). The prosodic structure, by contrast, follows the syntax closely and cannot ignore any syllable. Many prosodically different lines of text can instantiate one and the same meter. This shows that there are necessarily two, co-present prosodic structures in poetry (e.g. Blumenfeld 2015). This point can be demonstrated by considering the three possible prosodic phrasings that Selkirk (2000: 243) points out for our sample sentence.

Prosodic phrasings $(\mathrm{MaP}=$ major phrase $)$

a. (She lóaned her róllerblades to Róbin) $)_{\mathrm{MaP}}$

b. (She lóaned her róllerblades) $\mathrm{MaP}$ (to Róbin) $\mathrm{MaP}$

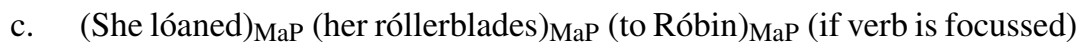

All phrasings can be fitted into the same, single metrical template. In each case the meter is invariant, whereas the regular prosody varies with syntax, morphology and information structure in the normal way. Another indication that there are two prosodic structures to respect is the fact that recitation of meter may vary between closer to the metrical schema (typical of the limerick and nursery rhymes), or closer to natural prosody (e.g. blank verse). The point that two structures are co-present in metered verse is in one sense trivial, and most general theories of meter also assume the presence of a metrical template. What is novel in our approach is the origin of that template in grammar, as a large prosodic morpheme, and the fact that grammatical constraints, thereby, can control meter directly.

The meter in (1) is iambic tetrameter (although the example was not constructed to illustrate a meter). It is important to note that the relationship between the metrical tree and the regular prosodic tree is one of homology, rather than correspondence. This brings us to the next point. If metrical templates are really just relatively unmarked prosodic structures, the question is what actually goes into them. Most models of meter assume that the metrical template comes with information relating to rhythm, often annotated as strong and weak positions (S/W), or as prestipulated headedness (iambic, trochaic, etc.). Line instances of text are then evaluated for grammaticality by the matching of linguistic prosody against the structure of the meter (Kiparsky 1977; Hayes and Kaun 1996; Hanson and Kiparsky 1996). The matching typically focuses on certain properties such as 'prosodic prominence to metrical position S', 'prosodic weight to metrical position S' or 'no prominence to metrical position $\mathrm{W}^{\prime}$.

In our model, where the metrical templates are constituted by prosodic domains drawn from the prosodic hierarchy, headedness comes about by the same set of UG constraints that govern ordinary language. Thus, templates are not prespecified for strong and weak positions. However, a meter may abide by constraints requiring prominences at edges of metrical domains. This comes about by alignment

\footnotetext{
${ }^{1}$ We will avoid the term 'metron' which is used in various senses and does not always refer to the same category in the prosodic tree, even within one and the same tradition (e.g. Greek iambics vs. dactyls).
} 
of prominence with some edge, via grammar. We shall look at examples of this in Sect. 2.1.

The important point here is that the way things are set up in our model, the notion of matching between meter and regular prosodic structure is not relevant, since the metrical template is not treated as an external object, but as a second, linguistic prosodic structure attached to the same text as the ordinary prosodic structure. This allows for grammar to intervene directly in the construction of verse, just as it does in more familiar prosodic morphology.

To supplant the notion of matching, we suggest instead that the metrical template is subject to the grammar in a particular and limited way, at least for some types of meter. The general idea is that a meter could require strict obedience to a particular constraint in the metrical tree, while allowing violation of the same constraint in the prosodic tree. We call such a constraint 'privileged'. We will illustrate how this works in Sect. 2.1 with meters from Spanish and Swedish before turning to Tashlhiyt Berber.

\subsection{Privileged constraints}

In the current approach there are two prosodic structures, regular prosody and meter, both of which relate separately to the constraints of grammar. The regular prosody is subject to the grammar in regular ways, of course, instantiating alignment with morphosyntactic categories, placing accents on tone-bearing units, dealing with rhythmic adjustment, etc. The shape of the metrical prosody (i.e. the metrical template) is partly the result of the grammatical wellformedness constraints as mentioned above (further discussed in Sect. 7). The question now is how this structure comes to express the other properties that are addressed by the stipulated S/W structure and matching in generative metrics, e.g. the common tendency for meters to have prominences primarily at the left or right edge. We suggest that this is due to the action of relevant constraints of grammar directly on the metrical structure. For example, if a language has left-alignment of stress in words as a matter of a phonological regularity, then this constraint may mandate left-alignment in the meter, too, since both prosodic structures contain the same prosodic categories. However, the status of the two prosodic structures differs in such a way that the outcome is not identical in the two structures. The regular prosodic structure is subject to faithfulness which means that lexical information (e.g. non-initial stress in some words) will overrule the desiderata of grammatical constraints (initial stress). In the meter, the action of such a constraint may be less hampered than in regular prosody, leading to a regularity that functions as part of the organisation of meter. This is the function of a privileged constraint.

We will illustrate this point with the example of the Spanish alexandrines of the Nicaraguan poet Rubén Darío (1867-1916) and the treatment of nuclear phrase accents (Piera 2001). ${ }^{2}$ In Spanish, phrasal prominence goes to the right edge of phrases. However, nuclear accents must occur on stressed syllables (=TBU), and stressed syllables are not always word-final. This means that not all phrase prominences will be strictly phrase final, in the regular prosody. In the meter, however, the generalization

${ }^{2}$ For a general discussion of Spanish nuclear accent, see e.g. Prieto (2006). 
is that all nuclear accents are perfectly aligned with the end of the halfline, i.e. they occur in positions 6 and 12. Post-nuclear syllables are extrametrical, in the meter. These points are illustrated in (3) and (4). Underlining marks synalepha. Syllables carrying nuclear accent are bolded and marked with an accent.

(3) Word-final accent on the 6th and 12th syllable (De otoño, 6) cuando empecé a crecér, un vago y dulce són...

$$
\begin{array}{lllllllllll}
1 & 2 & 3 & 4 & 5 & 6 / 7 & 8 & 9 & 10 & 11 & 12 /
\end{array}
$$

'... when I started to grow, a vague and sweet sound...'

(4) Extrametrical syllables following the 6th and 12th syllable (Tutecotzimí, 35) Es la mañana má $<$ gica $>$ del encendido tró $<$ pico $>$.

$\begin{array}{llllllllllll}1 & 2 & 3 & 45 & 6 & / & 7 & 8 & 9 & 1011 & 12 \quad /\end{array}$

'it is the magical morning of the tropic lightning'

In (3), nuclear accents occur in words with final stress, whereas in (4), they occur in words with nonfinal stress, a situation that leads to extrametricality in the meter. Thus, the beginning of the following halfline is suspended until the next word. ${ }^{3}$ In (5), we have the line in (4) annotated with prosodic structure above and meter below. The nuclear accent is marked as T* (a pitch accent, Piera 2001; Prieto 2006).

Alexandrine with nuclear prominences and extrametrical syllables

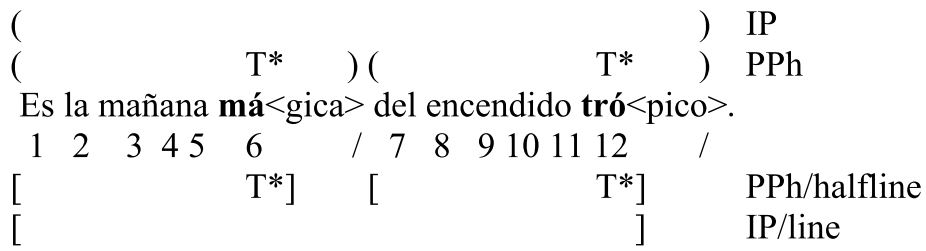

Let us first look at the regular prosody. The nuclear accent is on the stress of the phrase final word, but since this stress is not word-final, the nuclear accent is not flush at the right edge of the prosodic phrase. The prosodic phrase is well aligned with word edges. The constraints that lead to this result are the following.

$$
\begin{aligned}
& \text { Constraints for Spanish nuclear accent } \\
& \mathrm{TBU}=\dot{\sigma} \quad \text { 'the stressed syllable is the tone-bearing unit' } \\
& \text { ALIGN-R ( } \left.\mathrm{T}^{*}, \mathrm{PRPH}\right) \quad \text { 'every nuclear accent }\left(\mathrm{T}^{*}\right) \text { is at the right edge of a } \\
& \text { prosodic phrase' [privileged in meter] } \\
& \text { Align-R (PHPR, XP) 'every prosodic phrase is at the right edge of an XP' } \\
& \text { ALIGN-L (XP, PRPH) 'every XP is at the left edge of a prosodic phrase' }
\end{aligned}
$$

These constraints express the generalizations that nuclear accent occurs on stressed syllables at the right edge of prosodic phrases, and that prosody and syntax align.

\footnotetext{
${ }^{3}$ Single extrametrical syllables also occur, as do syllables without nuclear accent in position 6 . The various shapes of halflines combine freely (examples in Piera 2001).
} 
Crucial ranking in the grammar of Spanish

$\mathrm{TBU}=\dot{\sigma}$

Align-R (PhPR, XP) $\gg$ Align-R $\left(T^{*}\right.$, PRPH $)$
Align-L (XP, PRPH)

The ranking in (7) shows that the association of tones to TBUs and the alignment of prosodic and syntactic edges dominate the alignment of nuclear accent at the right edge of the prosodic phrase. As a result, nuclear accents will occur one or two syllables away from the right phrase-edge when stress isn't final in the accented word.

Turning to the meter, we find that the alignment of nuclear accents with the right edge of a prosodic phrase (i.e. halfline) is always perfect. We take this to be a defining principle of this meter. As seen in (5), the meter ends after a nuclear accent and simply disregards the unstressed syllables, which thereby become extrametrical in the meter. The proposal here is that the prosodic structure and the meter both employ the same grammar. The regular prosody does this in the familiar, linguistic manner, while the meter does this in very a limited manner, where it is sensitive to a particular constraint, the privileged constraint. Put differently, a meter could be defined by singling out a constraint as privileged, in this case ALIGN-R ( $\left.\mathrm{T}^{*}, \mathrm{PRPH}\right)$. That constraint should be dominated in the regular prosody, or else there could be no metrical improvement (reduction in markedness), but obeyed in the meter. ${ }^{4}$ In this sense, the meter provides overall grammatical improvement to the verse, expressing formally an often cited intuition. ${ }^{5}$ The improvement here resides in the fact that all nuclear accents are perfectly aligned in their domains, in the meter. This hypothesis about the way in which meter is constructed depends on the homology of regular prosody and meter.

One question that comes up at this point is what it means for a constraint to be privileged. One possibility is that the constraint is high-ranking in a metrical grammar, which is parallel to the linguistic grammar, where the same constraint would be lower in rank. This solution has some attraction but is very powerful in that it requires two grammars and therefore runs the risk of losing the connection between the individual language and the meter occurring in it. Another possibility, the one we entertain here, is that the privileged constraint is indeed singled out from the linguistic grammar by the poet, and that it is then made relevant to the meter tree, leaving other constraints invisible and irrelevant to that tree. ${ }^{6}$ It will not be possible to evaluate the predictions of these alternatives, or indeed to establish where predictions would differ for the two hypotheses, within the context of this article. For now we adopt the second hypothesis, i.e. that there is a mechanism, specific to meter, that selects a grammatical constraint and makes it the principle of the meter. This hypothesis seems also to be the closest to standard assumptions for meter, where the meter is seen as a reduced or stylized structure (e.g. a string of a specified length, perhaps

\footnotetext{
${ }^{4}$ Whether such a constraint should be obeyed absolutely or just more than in the regular prosody is an empirical question at this point. Possibly, more than one constraint could be privileged in a meter, though it would seem unlikely that they could conflict with each other.

${ }^{5}$ Marjorie Daunt (1946: 64) says for Old English that verse is "really the spoken language rather tidied up", and John Thompson (1961) expresses this as "language imitating itself".

${ }^{6}$ The constraints that shape the prosodic morpheme that constitutes the meter remain in effect, though, since that is a matter for the regular prosody.
} 
adorned with specifications for strong and weak positions), with limited relevance to grammar (e.g. matching of prominence/non-prominence to $\mathrm{S}$ or $\mathrm{W}$ ). The important point for our present purposes is the fact that the meter is under grammatical control, employing a grammatical constraint directly, rather than being subject to languageexternal mechanisms like matching. This puts meter more firmly under the purview of language.

The singling out of constraints as privileged allows authors to adopt different properties of a grammar in composing verse of ostensibly the same kind. A case in point is dactylic hexameter in Swedish (Riad 2013), where Esaias Tegnér and August Strindberg do just that. Esaias Tegnér (1782-1846), in his poem Nattvardsbarnen ('The communion children'), picks the constraint NOCLASH-PRWD 'Avoid clash within the prosodic word', which mostly targets compounds with adjacent stresses ('hän,ryckningen 'the rapture', 'hvit,menad 'whitewashed'). In the meter, this means that stress clash is avoided inside verse feet (=prosodic words), such that compounds are always split between verse feet. ${ }^{7}$ This in turn means that many word accents (on primary stresses) will occur to the right in verse feet, even though their typical location in simplex forms is to the left.

$$
\begin{aligned}
& \text { Tegnér, Nattvardsbarnen, lines 1-2 } \\
& \text { [Pingst, 'hän-][,ryckningens] [dag, var] [inne. Den] [landtliga] [kyrkan] } \\
& \text { [stod 'hvit-][,menad i] [morgonens] [sken. På] [spiran af] [tornet] } \\
& \text { 'Pentecost, rapture's time was here. The country church stood whitewashed } \\
& \text { in the morning glow. On the spire of the tower...' }
\end{aligned}
$$

For August Strindberg (1849-1912), in Trefaldighetsnatten ('Trinity eve'), dactylic hexameter admits clash within verse feet (=prosodic words), but instead mandates left-alignment of pitch accents in prosodic words, by ALIGN-L(T* PRWD). The relevance of this constraint in the language is easily seen in compounds where there are two or more stressed syllables (=TBU) and where the leftmost is the obligatory location of accent. In the language, ALIGN-L(T*, PRWD) is dominated by TBU $=\sigma^{\prime}$, such that we find many words with non-initial stress (bu'kett 'bouquet', harmo'ni 'harmony', nationali'tet 'nationality'). This resembles the Darío case we reviewed above, but concerns the level of the prosodic word, where accents are nearly obligatory (Myrberg and Riad 2015), rather than the prosodic phrase.

$$
\begin{aligned}
& \text { Strindberg, Trefaldighetsnatten, lines 112-113 } \\
& \text { ['Frun,timmers-][,veckan går] [in som] [damernas] [egen i] [juli] } \\
& \text { ['namns, dag var][eviga] [dag med] ['port,vin] [kaffe och] [doppa] } \\
& \text { 'Ladies' week begins like the ladies own in July nameday every day with port, } \\
& \text { coffee and buns for dipping.' }
\end{aligned}
$$

The result is two different linguistic principles for dactylic hexameter in Swedish, one where NoCLASH is privileged leading to instances of word accents that are mis-

\footnotetext{
${ }^{7}$ In the line-final verse foot, but only there, clashing spondees are considered legitimate, indeed desirable ('löf sal 'arbour', 'jern ,kors 'iron cross'), presumably as an emulation of the final quantitative spondee of classical hexameter.
} 
aligned with left edges of verse feet (=prosodic words), i.e. Tegnér, and one where word accents are always aligned with left edges of verse feet, and where clashes within verse feet (=prosodic words) are common, i.e. Strindberg. Both of the constraints that are privileged in the two types of hexameter here are dominated in the ordinary language grammar. Thus, the language tolerates clashing compounds as well as word accents that are not word-initial.

The meters that we have looked at, and also Tashlhiyt (below), all exhibit constancy with regard to features that are recognizably linguistic. Thus, the placement of prominence is constant in Darío's alexandrines and Strindberg's hexameter, and the avoidance of clash is constant in Tegnér's hexameter and the Tashlhiyt straight meters. This constancy allows us to partly define the meters in terms of a privileged constraint, which makes the boundary between grammatical and ungrammatical clear for a meter. Other systems, such as the English iambic pentameter, exhibit much less constancy, or at least the constant features are much less in the foreground. To the extent that such meters are characterized by gradient wellformedness, which is the general tendency in analyses of it, approaches employing matching may attain a more fine-grained analysis. For instance, the faithfulness approach of Blumenfeld (2015) allows for a rather complete evaluation of the wellformedness (i.e. degree of faithfulness between meter and regular prosody) of individual lines of poetry, cf. (55). A discussion of the implications for typology will have to be left aside here.

We now turn to the straight meters of Tashlhiyt Berber. We shall find that, while many things are different, this quantitative meter is most similar to Tegnér's dactylic hexameter in that it employs NOCLASH as its privileged constraint. While all individual meters obey NoCLASH (and to some extent NOLAPSE), they must also be specified in some way in order to be different from each other. This entails markedness. I argue that the lexical fixation of individual meters, primarily regarding their length, is best expressed as distinctive markedness, i.e. as underlying forms containing required violations of constraints (Golston 1996). We discuss this briefly in Sect. 7.3. The upshot is that line length emerges from grammar via unmarked binarity, but combines with markedness on binarity constraints to yield their different lengths.

\section{Tashlhiyt meter-basic observations and previous scholarship}

We begin by establishing the regulation of syllable number and syllable quantity observed by Jouad $(1993,1995)$. Below is an excerpt from the song called Ndalb irbbi in the transcription provided by Dell and Elmedlaoui (2008: 7ff.). The vertical lines indicate the grouping proposed and defended by Dell and Elmedlaoui (2008). The metrical line constitutes the syllabification domain. Syllable weight in each position is indicated with L for 'light' (CV, CC), and H for 'heavy' (CVC, CCC). Syllables with a geminate in their coda may be treated as $\mathrm{H}$ or $\mathrm{L}$, depending on what fits the meter, as long as the geminate straddles the syllable boundary (so-called 'hinge codas', Dell and Elmedlaoui 2008: 57). The few syllables that do not correspond to the weight indicated are bolded. In the transcription, marks for pharyngealization and labialization are omitted. 
(10) Ndalb irbbi, stanza-initial lines

\begin{tabular}{|c|c|c|c|c|c|c|c|c|c|c|c|c|c|}
\hline & 2 & 3 & 4 & 5 & 6 & 7 & 8 & 9 & 10 & 11 & 12 & 13 & \\
\hline & $\mathrm{H}$ & $\mathrm{I}$ & I & $\mathrm{L}$ & $\mathrm{H}$ & $\mathrm{L}$ & $\mathrm{L}$ & $\mathrm{L}$ & $\mathrm{L}$ & $\mathrm{L}$ & $\mathrm{L}$ & $\mathrm{H}$ & \\
\hline 1 & $a-$ & ha- & la & ha & yah & ni & na & wa- & li & ya & ві & $\mathrm{lq}$ & \\
\hline 2 & dal & $\mathrm{ba}$ & $\mathrm{ka}$ & di & yit & $\mathrm{sa}$ & $\mathrm{ra}$ & tl & $\mathrm{xi}$ & $\mathrm{a}$ & fu & snn & \\
\hline 3 & $\operatorname{mnx}$ & $\mathrm{S} 1$ & n & ga & win & $\mathrm{ku}$ & ri- & li & ma- & yi & $\mathrm{kn}$ & uf & \\
\hline 4 & $\mathrm{a}$ & $\mathrm{Z}$ & $\mathrm{a}$ & $\mathrm{da}$ & кі & 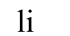 & $\mathrm{nb}$ & ві- & li & $\mathrm{sr}$ & B1 & slh & \\
\hline 5 & dur & na & si- & $\mathrm{dl}$ & lu & la & $\mathrm{nl}$ & la & ви & sa & $\mathrm{ca}$ & $\Pi$ & \\
\hline 6 & $\mathrm{u}$ & ya & & sas & tll & Sa & ru- & ra- & $\mathrm{km}$ & a & $\$ 1$ & ix & \\
\hline 7 & ma- mun & ra & & $\mathrm{da}-$ & lit & ma- & $\mathrm{mu}$ & ri & ва & ва & $\mathrm{ra}$ & snn & \\
\hline 8 & hu & ya & ta- & ga & tll & ৎa & ru- & ra- & $\mathrm{km}$ & na & S1 & ix & \\
\hline & wa- & na & $\mathrm{r}$ & gi & вit & 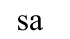 & $\mathrm{sm}$ & ha & кі & wa & wa & lr & \\
\hline & 1 & $\mathrm{dl}$ & & bu- & ratt & ni- & tu & ya & na & ri & $\mathrm{sm}$ & $\mathrm{mu}$ & \\
\hline & $\operatorname{mim}$ & wa & $\mathrm{u}$ & $\mathrm{dn}$ & $\mathrm{kal}$ & xi & ri- & fu & ви & ва & $\mathrm{ra}$ & & \\
\hline & $\mathrm{ma}$ & $\mathrm{ka}$ & ttt & 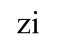 & yan & $\boldsymbol{a}$ & $\mathrm{ru}$ & wa- & li & k1 & & & \\
\hline 4 & dal & bi & & bi- & lan & ьі & $\mathrm{xl}$ & qn & fi & na & Bl & $\mathrm{aq}$ & \\
\hline 15 & wrn & ga & & & 111 & ৎa & $\mathrm{ru}$ & la & sa & rn & sa & & \\
\hline & ix & wa & $\mathrm{mu}$ & & kal & ৎa & $\mathrm{rx}$ & šn & ni & ва & $\mathrm{ra}$ & nn & \\
\hline
\end{tabular}

(11) Ndalb irbbi, refrain

$\begin{array}{lllllll}1 & 2 & 3 & 4 & 5 & 6 & 7 \\ \text { L } & \text { H } & \text { L } & \text { L } & \text { L } & \text { H } & \text { L } \\ \text { n } & \text { dal } & \text { bi } & \text { rb } & \text { bi } & \text { slY } & \text { fu }\end{array}$

Translation (including the refrain for the first two lines)

1 Allah! Allah! O merciful who has created us, I'm begging God for my salvation.

2 I pray you, let your hand grant me its benefactions. I'm begging God for my salvation.

3 I believe in you, I am yours, Nothing is better for me than you.

4 Allow me to make a living by licit means.

5 So that I never live in humiliation, nor be on the path leading to it.

6 No, skein of evil! I shall not carry you on my head.

7 What do I have to do with humiliation? What will I look for on the path leading to it?

8 No, bundle of evil! I shall not carry you on my head. [9=7]

10 Those who try to make fun of me, I will not listen to their talk.

11 For only when one dies does one forget good and evil.

12 Your seeds are sweet, O goodness, and your path is not a dead end.

13 How could one scold those who put you (i.e. goodness) in what they say?

14 I beg God who has created me and endowed me with reason.

15 Let me not do evil nor say evil. [16-17 =6-7]

18 Your seeds are bad, $\mathrm{O}$ evil, and so is your path. [...]

In this particular meter, each line has fourteen syllables. The refrain has seven syllables and we leave it to the side for now. The last syllable of each line contains a so-called stopgap vowel (marked '[i'), which is a device that improves the text-to- 
tune alignment, ostensibly by syllabifying a line-final consonant. When medial, such a vowel carries a metrical function, while when used at the end of a line, like here, it does not (Dell and Elmedlaoui 2008: 63ff.). The quantitative distribution of syllables within each line is the same. Thus, the first group of syllables is LHL, the second is LLH, the third LLLL, and the fourth LLH. In each line this quantitative distribution is repeated, with few exceptions (some of which are licenses). Dell and Elmedlaoui refer to the units in this grouping as 'feet', and this should be understood as a verse unit. In order to distinguish this unit from prosodic feet, but remain close to Dell and Elmedlaoui's term, I shall refer to them as 'verse feet'. ${ }^{8}$ The verse feet are constituted by two metrical positions (H or LL) which are homologous to prosodic feet (Golston and Riad 2000, for Greek).

This poetic tradition is constituted by songs, which means that there is a musical structure, including rhythm, beside the metrical structure. There has been some discussion regarding the relationship between the two, e.g. regarding English folk songs (Hayes and MacEachern 1998; Kiparsky 2006). The issue has been whether the rhythmic properties of the metrical structure can be reduced to those of the musical structure or not. With Tashlhiyt Berber, it is quite obvious that the two structures are separate, as also argued by Dell and Elmedlaoui (2008: 23ff.). The regular alignment of verse feet with the musical rhythm targets the second mora of each verse foot, which coincides with the first downbeat of the bar. The second mora of the verse foot may be the weak or strong mora of a prosodic foot, which we take to be the moraic trochee [ $\left.{ }^{\prime} \mu \mu\right]$, realized as [ $\left.{ }^{\prime} \mathrm{LL}\right]$ or $\left[{ }^{\prime} \mathrm{H}\right]$. It is the weak mora in LLH and (presumably) the strong in LHL. ${ }^{9}$ There is alternation of verse feet that is internal to the metrical structure (see (15)), and which has nothing to do with the rhythm of the music. Also, there is no direct connection between the weight of syllables and the duration of notes (Dell and Elmedlaoui 2008: 44). More broadly, speakers of Tashlhiyt Berber can pass metricality judgements on the wellformedness of a meter, regardless of the presence of music (2008: 30). ${ }^{10}$ Our analysis of the meter further shows that the meter contains properties (clash avoidance, final lapse avoidance) that make no sense in terms of musical rhythm. In (12) the caret marks the tactus, i.e. the beat of the music.

\footnotetext{
${ }^{8}$ One could equally call them as 'metra', which would be in greater parallel with the common usage of terms for Greek and Latin metrics.

${ }^{9}$ The distinction between prominent and non-prominent moras, following from the prominence structure of the moraic trochee (Kager 1993), is important for the analysis given in this article. However, I make no claims regarding Tashlhiyt stress and intonation. Dell and Elmedlaoui (2002: 14) observe that there appears to be phrase-level prominence on final or penultimate syllables, and Gordon and Nafi (2012) find evidence for high pitch at the end of the intonation phrase, as well as intensity peaks on word-final syllables. The latter is interpreted as word stress, which is, however, much less conspicuous than the pitch movements at phrase boundaries. Realization patterns of pitch peaks are studied in Grice et al. (2015).

${ }^{10}$ The alignment of the second mora of verse feet to the downbeat of the music and the absence of verse feet beginning with $\mathrm{H}$ might still look like a potential, intriguing connection of facts. In the present analysis, however, the absence of verse feet beginning with $\mathrm{H}$ (i.e. HH and HLL) follows from the general constraint against clash that appears to guide several other aspects of the meter.
} 
Alignment of meter with musical structure (Ndalb irbbi)

.$=108$

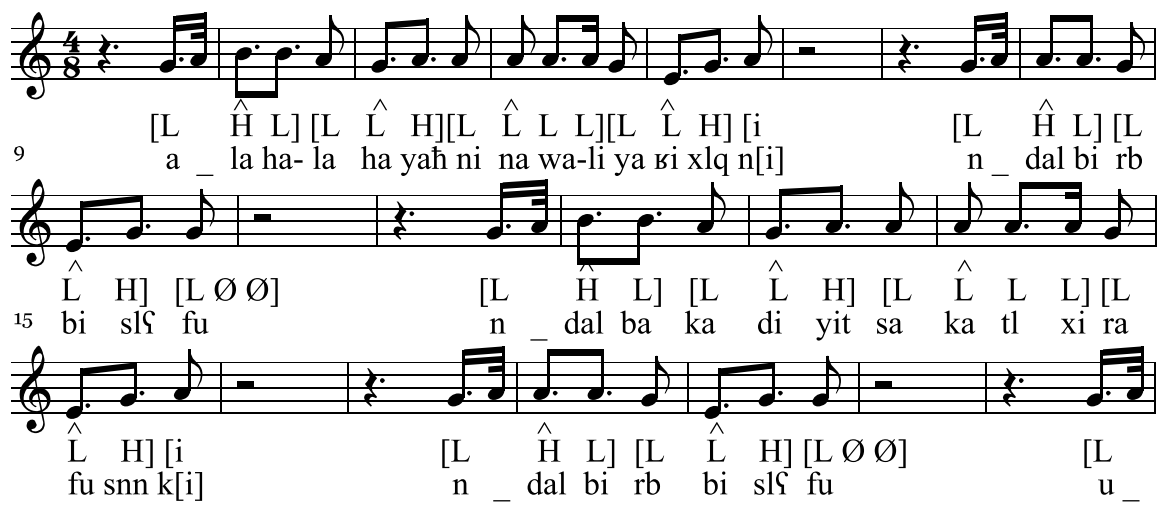

Metrical patterns may combine to make stanzas, which in Dell and Elmedlaoui's terms refer to a stretch of text corresponding to a melodic round, the whole song being made up of melodic rounds drawn from a small set of sub-tunes ( 1 or 2 , mostly). The text of a stanza is also associated with a specific metrical content, i.e. a metrical line. These lines may be constituted by a single meter (what we will call an 'independent meter'), or a combination of meters (either independent or 'dependent meters' or combinations thereof). The beginning of Ndalb irbbi in (12) above is constituted by two stanzas made up of two independent meters (2B-a+1A-c, see (10), (11) and (25)). There are many meters but they are all closely related to each other, as we shall see.

In what follows we will focus on the internal structure of the meter, and only comment on the musical structure where it appears to cast light on the meter.

\subsection{L4m, classes and Altern}

Dell and Elmedlaoui summarize the common properties of the structure of the Tashlhiyt Berber verse foot with the formula ' $\mathrm{L} 4 \mathrm{~m}$ '. This formula expresses the fact that verse feet always begin with $\mathrm{L}$ and that they contain 4 moras. These conditions limit the set of possible verse feet considerably.

$$
\begin{array}{ll}
\text { Legitimate and illegitimate verse feet, L4m } \\
\{\text { LHL, LLLL, LLH }\} & \\
*\{\text { HH, HLL }\} & \text { begin with } \mathrm{H} \\
*\{\text { LHLL, LLLH, LH, LLL }\} & \text { more or fewer than } 4 \text { moras }
\end{array}
$$

L4m neatly summarizes central generalizations of the straight meters of Tashlhiyt Berber but does not actually provide an answer to the question why things should be this way. Why can't verse feet begin with an a H syllable? What motivates the constraint on four moras?

The legitimate verse feet fall into two classes that Dell and Elmedlaoui call ' $O$ ' (odd) and ' $E$ ' (even), where the terms refer to whether the heavy syllable occurs in an odd or even position, counting from the left. The classes are motivated by the way verse feet alternate, i.e. the Tashlhiyt straight meters are all mixed. 


$$
\begin{array}{ll}
\text { Classes of verse feet } & \\
\mathrm{O}=\{\mathrm{LLH}\} & (\mathrm{H} \text { in } 3 \mathrm{rd} \text { position, odd }) \\
\mathrm{E}=\{\mathrm{LHL}, \mathrm{LLLL}\} & (\mathrm{H} \text { in } 2 \text { nd position, even })
\end{array}
$$

The alternation is very stereotypical and formulated as the condition ALTERN, in Dell and Elmedlaoui (2008: 39).

Altern: Adjacent verse feet do not belong to the same class, E or O

Thus, successive verse feet will alternate between the two classes. The basic property that puts LHL and LLLL in the same class becomes evident when we look at the segmentation, where LLLL is L.LL.L, in parallel with L.H.L. This somewhat unexpected property is motivated in Dell and Elmedlaoui's study by arguments concerning distribution and patterning under catalexis.

In his ground-breaking study, Jouad (1995: 333) made the observation that one never finds two heavy syllables next to each other in the meter. ${ }^{11}$ Dell and Elmedlaoui formulate this as the condition $\mathrm{NoHH}$ (2008: 59).

(16) NoHH: No Tashlhiyt or Tamazight meter can require lines to contain adjacent $\mathrm{H}$ syllables

In fact, the NoHH condition follows from the formulation of $\mathrm{L} 4 \mathrm{~m}$. If all verse feet contain four moras and none can begin with $\mathrm{H}$ then there will never be any pair of adjacent H's in the meter under any sequencing of verse feet admitted by L4m. But, as analyses, neither NoHH nor L4m are satisfactory, since they express the generalization without indicating a basis for it.

In the main argument of this article, I pursue an analysis of the observations made by Jouad and Dell and Elmedlaoui which aims at an understanding of the straight meters in terms of the linguistic grammar of Tashlhiyt Berber, in the light of the general relationship between meter and grammar proposed in Sect. 2.

\section{Tashlhiyt Berber verse feet}

Let us now look at the properties of the Tashlhiyt Berber verse feet. The unmarkedness of binarity is highly visible in verse feet. In the natural class of meters that Dell and Elmedlaoui call 'straight', all verse feet contain four moras (unless catalectic at the edges of lines). In terms of prosodic structure this amounts to binary prosodic feet (=metrical positions), pairwise organised into binary verse feet. This is distinct from e.g. Greek and Arabic, both of which systems contain some meters that have metrical positions that contain unary prosodic feet $(\mathrm{L})$. The starting point for the possible verse feet in Tashlhiyt Berber, then, is four moras.

The distribution of these moras in syllables entail rhythmic properties that challenge rhythmical constraints differently. The prosodic foot of Tashlhiyt Berber is assumed to be the so-called moraic trochee (McCarthy and Prince 1986; Hayes 1987, 1995; Kager 1993). This is the prosodic foot for quantitative systems like e.g. Arabic,

11 "Dans une formule étalon, deux unités lourdes [... ] ne se suivent jamais" (Jouad 1995: 333). 
Greek, Latin, and a host of other languages (Hayes 1995). The prominence profile of the moraic trochee is [ $\left.{ }^{\prime} \mu \mu\right]$, where the first mora is prominent and the second nonprominent (Kager 1993). In syllabic terms this comes out as ['LL] or ['H].

Below we list the set of available verse feet containing the unmarked number of four moras together with their rhythmic properties at the syllable level and the mora level.

\begin{tabular}{|c|c|c|c|c|}
\hline & Shape & $\begin{array}{l}\text { Syllabic } \\
\text { prominence }\end{array}$ & $\begin{array}{l}\text { Moraic } \\
\text { prominence }\end{array}$ & $\begin{array}{l}\text { Rhythmic } \\
\text { property }\end{array}$ \\
\hline dactyl & H LL & $\underline{\mathrm{XX}}$ & $X . X$. & clash \\
\hline spondee & $\mathrm{H} \mathrm{H}$ & $\overline{\mathrm{xx}}$ & $\mathrm{X} . \mathrm{X}$. & $\overline{\text { clash }}$ \\
\hline proceleusmatic & LL LL & $\mathrm{X} \cdot \mathrm{X}$. & $X \cdot X$. & - \\
\hline anapest & LL H & $\mathrm{x} \cdot \mathrm{x}$ & $\mathrm{X} \cdot \mathrm{x}$. & - \\
\hline amphibrach & L H L & . $\mathrm{x}$. & . $\mathrm{x} \ldots$ & lapse \\
\hline "resolved amphibrach" & L LL L & . $\mathrm{x} \ldots$ & . $\mathrm{x} \ldots$ & $\overline{\text { lapse }}$ \\
\hline
\end{tabular}

The unshaded verse feet are the ones we find in Tashlhiyt Berber, while the shaded ones are excluded. The dactyl and the spondee entail a clash. These are used in Greek hexameter, but are banned from Tashlhiyt straight meter. The anapest and the proceleusmatic occur together with the dactyl and spondee in Greek anapestic verse. In such verse, the proceleusmatic is very rare and only occurs in song (Raven 1962: 56). In Tashlhiyt straight meter, every other verse foot is an anapest, but the proceleusmatic is absent. The resolved and unresolved amphibrachs are frequent in Tashlhiyt meter, but completely absent in Greek meter.

The table in (17) evaluates abstract verse feet rather than the prosodic structure of words. Based on the discussion in Sect. 2, we assume that any line of metrical poetry comes with two co-present prosodic structures, one of which is the meter, and the other the regular linguistic prosodic structure.

As far as rhythm is concerned, the trend in the straight meters is for Tashlhiyt verse feet to avoid clash (dactyl, spondee). There is no particular rhythmic reason why the proceleusmatic should be excluded. ${ }^{12}$ At any rate, of the verse feet cited in (14), one class contains the unmarked four-mora verse foot $\{$ LL.H $\}$, the prototypical anapest, and the other class contains precisely the two types of four-mora verse feet that contain a lapse $\{$ L.H.L, L.LL.L $\}$.

The anapest LL.H is made up of two prosodic feet ['LL] and ['H] (moraic trochees). In the amphibrachs the shape of the verse feet does not admit a clean division into two prosodic feet, i.e. L.H.L and L.LL.L, though the four-mora count is intact. This is the situation that is traditionally called 'anaclasis', i.e. the substitution of place of a $\mathrm{H}$ and a $\mathrm{L}$ syllable. In Greek meter anaclasis is not tolerated except for a few cases found in some lyric lengths (Halporn et al. 1980: 23). Greek admits lapses,

\footnotetext{
${ }^{12}$ One could imagine that the proceleusmatic occurred in the same class as the anapest, i.e. $\{$ LL.H, LL.LL\}, but possibly it is too surface similar to the resolved amphibrach (L.LL.L), which forms a class with the amphibrach \{L.H.L, L.LL.L\}. For the purposes of this article, we will rule it out by a requirement that final prosodic feet in prosodic words be heavy, see (23).
} 
but those lapsing structures always involve a monomoraic metrical position (L). ${ }^{13}$ In Tashlhiyt, however, monomoraic positions can only occur at the edges of a meter. Line-internally, verse feet are always four moras. Anaclasis in Tashlhiyt involves the positional substitution of a prosodic foot, $\mathrm{H}$ or LL, with a L syllable. One of the central questions regarding the Tashlhiyt meter system is to understand what motivates the shape of these verse feet.

The main hypothesis that I will defend is that rhythmic concerns are central in Tashlhiyt Berber metrics. The abstract template for the straight meter can be characterized in terms of how it obeys and violates rhythmic constraints that are relevant in language.

\subsection{NoClash, NoLapse and PrWd-Final H}

I propose that the fact that we do not find two adjacent heavy syllables (NoHH, Jouad 1995) is due to the linguistic constraint against clashing prominences, NoCLASH (Prince 1983).

\section{NoCLASH: Avoid adjacent prominent syllables}

Two adjacent $\mathrm{H}$ syllables will entail a clash, and that is a situation that Tashlhiyt Berber metrics clearly does not tolerate, as per (18).

If (18) is right for Tashlhiyt Berber, the sequencing of ['H] followed by ['LL] should also be excluded, since that dactylic configuration contains a clash, too. This is correct for the straight meters, within as well as across verse feet, as can be easily ascertained by looking over the tables in (25) and (26) below. We can thus make a broader generalization for the straight meters of Tashlhiyt Berber by (18), than what can be had by (16), and it means that clash avoidance is a major concern in this system.

In fact, the generalization is even wider, as we can also derive the very alternation of verse feet from the constraint NoCLASH. This is illustrated in (19), where syllabic prominences are marked on the first mora of each canonical prosodic foot. Dots mark the boundaries of prosodic units within the verse foot. This is the same meter as that given in (10) above.

Ndalb irbbi syllabic prominence structure

\begin{tabular}{|c|c|}
\hline & $\mathrm{X}$ \\
\hline .'H.L & 'LL.'H \\
\hline
\end{tabular}

This sequence, like all other sequences in the corpus analyzed by Dell and Elmedlaoui, contains no clash. In each transition, be it within a verse foot or across verse feet, there is always a non-prominent syllable between prominent positions. Given the requirement of four-mora verse feet, this can only be achieved if there is alternation of verse foot shapes. To illustrate this fact, consider a line of meter consisting of solely the most unmarked anapestic foot, LL.H.

\footnotetext{
${ }^{13}$ In trimeter lapses happen across verse feet (i.e. L.H L.LL, L.LL L.H), while in some lyric meters we find true trochaic, lapsing verse feet (․․), cf. Golston and Riad (2000, 2005).
} 
Clashing meter

$$
\text { 'LL. 'ㅆ 'LL.' } \underline{H} \text { 'LL. ' } \underline{H} \text { 'LL.' } H
$$

This meter does occur in Tashlhiyt Berber, but is not part of the straight meters (Dell and Elmedlaoui 2008: 102f.). An anapestic tetrameter yields three instances of clash across verse feet (indicated by the underlining in (20)). The proposal here, then, is that the alternation of verse feet in the straight meters has a rationale under clash avoidance. ${ }^{14}$

The very shape of the lapsing verse feet $\{$ L.H.L, L.LL.L $\}$ can thus be derived from clash avoidance, given unmarked LL.H as our starting point. Only if every other verse foot is either L.H.L or L.LL.L is clash fully avoided across a line. No other profiles of four-mora verse feet avoid clash in combination with LL.H.

This argument relies on a particular interpretation of the prominence profile of L.H.L and L.LL.L, as lapsing, see (17). These structures contain anaclasis, a phenomenon that may look straightforward in terms of syllables: LL.H comes out as L.H.L by switching the places of the last two syllables. However, since the middle position in an anaclastic verse foot can also be LL, it is hardly the question of a switch of syllables. Rather, the last prosodic foot is displaced one mora to the left. In terms of prosodic feet and moras, anaclasis causes the unmarked structure [' $\mu \mu]\left[{ }^{\prime} \mu \mu\right]$ to show up as the marked structure $\left[\mu\left[{ }^{\prime} \mu \mu\right] \mu\right]$. One prosodic foot is thus broken up $([\mu \ldots \mu])$ and the other prosodic foot remains intact $\left(. .\left[^{\prime} \mu \mu\right] \ldots\right)$. I take the rhythmic structure of the broken prosodic foot to be affected such that no prominence occurs on its first mora. The internal reason for this is the fact that, if the prominence would remain on the initial mora, a clash would occur between the first two moras of the verse foot $\left.\left({ }^{*}{ }^{\prime} \mu\left[{ }^{\prime} \mu \mu\right] \mu\right]\right)$, and nothing would be gained by anaclasis. The general reason would be that a single, stranded L syllable cannot be prominent, i.e. degenerate feet are not prominent in this language. The inner foot is intact, and so it retains its prominence.

Our analysis derives Dell and Elmedlaoui's condition ALTERN from clash avoidance. It is thus not a question of a mixed meter in any deep sense. The alternation is always the same in the straight meters, unlike the case in e.g. mixed Greek Aeolic meters. Rather than stipulating the alternation, the shape of verse feet and their combination are jointly driven by unmarkedness (four moras to the verse foot) and absolute clash avoidance.

The other rhythmic constraint, NOLAPSE, is also involved in shaping Tashlhiyt Berber meter, and it has two versions.

NOLAPSE- $\mu / \sigma$ : Avoid adjacent non-prominent moras/syllables

A lapse is defined in moraic and syllabic terms. The distinction appears to play a small role in Tashlhiyt meter. The final sequences of the anaclastic, amphibrachic verse feet \{L.H.L, L.LL.L $\}$ entail moraic lapses, and the resolved amphibrach L.LL.L in addition entails a syllabic lapse, see (17). Thus, a sequence of two non-prominent

\footnotetext{
${ }^{14}$ Dell and Elmedlaoui (2008: 102f.) cite the meter in (20) as an example of a meter which violates their condition ALTERN. (They also entertain the alternative analysis as $\varnothing$. .L L.H.L L.H.L L.H.L L.H., , but discard it as it does not comply with the normal second-mora alignment with the downbeat in the music.) Its existence shows that the system is not exhausted by the meters we are looking at here. The proposals here_as in Dell and Elmedlaoui (2008) — are restricted to the natural class of straight meters.
} 
moras entails violation of NOLAPSE- $\mu$, and sometimes of NOLAPSE- $\sigma$. Whenever there is no reason to distinguish between moraic and syllabic lapse, we will simply refer to the constraint as NOLAPSE.

Lapses are frequent and occur in every other verse foot, as occasioned by the strict adherence to the dominating NoCLASH constraint. This does not mean that NOLAPSE is gratuitously violated, i.e. violation is minimal (Prince and Smolensky 1993). NOLAPSE is clearly visible in the absolute prohibition against lapsing verse feet at the end of a meter. ${ }^{15}$ Lines can end in amphibrachs as long as they are catalectic (L.H.Ø, L.LL.Ø, L.Ø.Ø), whereas acatalectic amphibrachs are systematically absent in that position (see Sect. 5.1 below). An understanding of this fact, which we will refer to as 'endlapse', can be had if we consider the influence of NOCLASH on verse foot distribution. A line-medial amphibrach avoids clash in both directions, whereas a line-final one only does so to the left. This makes the lapse of a final acatalectic amphibrach incur a gratuitous violation of NOLAPSE, as illustrated in (22).

Moraic prominence structure and endlapse

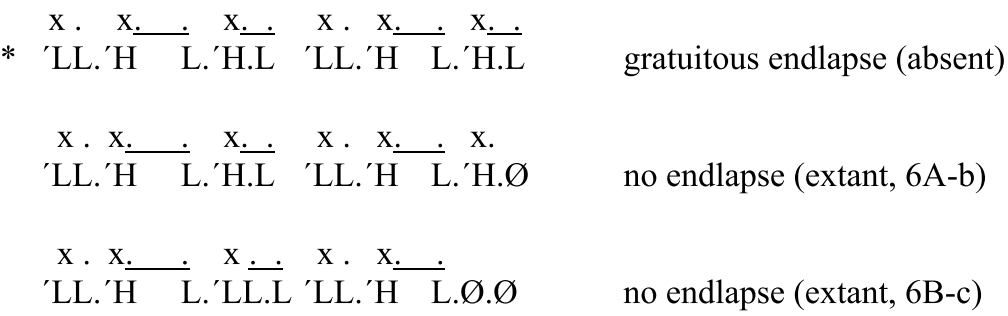

We thus attribute the absence of lapsing acatalectic verse feet at the end of lines to the regular grammatical workings of NOLAPSE in a position where NOCLASH is not challenged. Final catalectic amphibrachs are still generated, however, a fact that we attribute to unmarked binarity (Sect. 6.2). Note that the surface line may contain a lapse towards, or right at, the end, as long as it is driven by obedience to NoCLASH. It is when the lapse becomes gratuitous that it is prohibited, by NOLAPSE. This is the regular operation of grammar on the metrical template.

Another indication of the workings of NOLAPSE is seen in the prohibition against the resolved amphibrach (L.LL.L) line-initially (Dell and Elmedlaoui 2008: 86). We might analyze this as due to the higher liability of a syllabic lapse (which entails a moraic lapse), compared to just a moraic lapse. This makes L.H.L preferable over L.LL.L.

We need one more constraint to fully delimit the set of occurring verse feet in the straight meters. Some factor militates against the proceleusmatic (LL.LL). It is not common in Greek meter and it is absent in Tashlhiyt and Arabic meter. Rhythmically it is even better than the anapest, since it entails no clash or lapse within or across verse feet. But there must be other drawbacks, since it is so rare. One issue is that it only contains light syllables, whereas the language also has heavy syllables.

\footnotetext{
${ }^{15}$ The workings of NOLAPSE here actually concerns the stanza rather than the line of meter. Dependent meters may end in acatalectic lapsing verse feet (see Sect. 5.6) and we take this to be an indication of how they may combine with other meters (i.e. before rather than after), though this point needs to be empirically validated.
} 
In a potential Tashlhiyt meter, the proceleusmatic would only ever be useful as an alternant to the anapest LL.H, much like the resolved amphibrach L.LL.L is an alternant to the amphibrach L.H.L. Another problem is the very presence of L.LL.L. There could be problems with having two verse feet shaped LLLL in the same meter. Neither of those problems is linguistic in nature. What is linguistic is the fact that prosodic domains tend to end in a heavy syllable. At the phrase level, languages may have phrase final lengthening, a rule that is often taken to be the linguistic backdrop to the requirement of heavy syllables at the end of meter, or the related license for brevis in longo (Raven 1962: 26, Fabb 2002: 175). The same tendency can be found also in smaller domains, such as the prosodic word (i.e. verse foot). Arabic meter has this as a tendency (Golston and Riad 1997). While there is no knowing at this stage exactly what conditions the absence of the proceleusmatic in Tashlhiyt, we will suggest a constraint along these lines as responsible for the preference of LL.H over LL.LL. ${ }^{16}$

PRWD-FINAL H: Prosodic words (=verse feet) end in a heavy syllable

This alignment constraint requires verse feet to end in a monosyllabic metrical position, which must be $\mathrm{H}$ given that metrical positions are prosodic feet. The constraint PRWD-FINAL H is violated under pressure from privileged NOCLASH. If (23) is on the right track, we can understand why there is variation between $\mathrm{H}$ and LL only in case this prosodic foot is non-final in the verse foot (i.e. in L.H.L/L.LL.L).

NoCLASH and binarity narrow the shape of verse feet down to LL.H and either of L.H.L and L.LL.L. We now turn to the 56 straight meters that employ these verse feet.

\section{Line shapes in Tashlhiyt Berber metrics}

In this section we look at the shapes of the meters collected by Dell and Elmedlaoui (2008: 82ff.). The purpose is to provide a sense of the metrical space circumscribed by this corpus and how the variation comes about. Methodologically, this involves looking at resemblances and differences between meters, but also noting what potentially expected meters are missing. One of the most important ways in which meters vary is line length, and the system regulates single positions at either line end by catalexis. Below, we look first at how catalexis patterns at the line edges (Sect. 5.1) and then turn to the independent meters (Sect. 5.2). These are the 35 meters that can form stanzas alone, which amounts to them being able to fit into a musical line containing four beats. ${ }^{17}$ Dell and Elmedlaoui call these the 'self-sufficient' meters, the other class being the 'non-self-sufficient' meters. We will use the less cumbersome terms 'independent' and 'dependent' meters.

\footnotetext{
${ }^{16}$ Whatever it is that is wrong with LL.LL, it should reside in the shape of the verse foot. There would be problems pertaining to FIT (Hanson and Kiparsky 1996) if a line contained no $\mathrm{H}$ syllables, but in view of the fact that L.H.L and L.LL.L alternate with each other, it can't really be a requirement on all verse feet to contain a $\mathrm{H}$.

${ }^{17}$ Dell and Elmedlaoui (2008: 82ff.) indicate this with a divide in their tables, the shortest independent meters containing three verse feet (with some catalexis).
} 


\subsection{Catalexis}

Catalexis in Tashlhiyt Berber meter only applies at the edges of the line, where it provides some evidence for the class affinity of L.H.L and L.LL.L. Catalexis may remove one or two syllables, and it applies in such a way that the middle metrical position of L.H.L and L.LL.L is treated as a unit. Thus, while catalexis normally targets a single syllable at a time (irrespective of metrical position), the middle LL of L.LL.L is never split by catalexis.

Catalexis in verse feet

\begin{tabular}{|c|c|c|c|}
\hline & Initially & Finally & \\
\hline \multirow[t]{3}{*}{ LHL } & L.H.L & absent & acatalectic \\
\hline & $\varnothing . H . L$ & L.H. $\varnothing$ & catalectic \\
\hline & $\varnothing . \varnothing . L$ & L.Ø.Ø & catalectic \\
\hline \multirow[t]{3}{*}{ LLLL } & $\operatorname{absent}^{18}$ & absent & acatalectic \\
\hline & & L.LL. $\varnothing$ & catalectic \\
\hline & & L.Ø.Ø (*L.L $\varnothing . \varnothing)$ & catalectic \\
\hline \multirow[t]{3}{*}{ LLH } & LL.H & LL.H & acatalectic \\
\hline & $\varnothing \mathrm{L} . \mathrm{H}$ & LL. $\varnothing$ & catalectic \\
\hline & $\varnothing \varnothing . H$ & LØ.Ø & catalectic \\
\hline
\end{tabular}

The evidence for the dependence of L.LL.L on L.H.L is indirect in that *L.L $\varnothing . \varnothing$ is not attested. The shapes L. $\varnothing . \varnothing$ and $\varnothing . \varnothing . L$ could thus always be analyzed as the catalectic variants of L.H.L.

Dell and Elmedlaoui also show that L.H.L has freer distribution than does L.LL.L, in that no independent meter begins with L.LL.L. Also, catalectic patterns in meters of equal length tend to occur to a greater extent with metrical patterns containing L.H.L than those containing L.LL.L. These distributional facts lead us set up L.H.L as more preferred internally to the class. We have attributed this asymmetry to markedness in terms of moraic and syllabic lapses in (17), above, which would predict that L.H.L should be preferred over and above L.LL.L, all things being equal. ${ }^{19}$

\subsection{Independent meters}

The meters are cut from the same cloth, i.e. form a natural class, as clearly indicated by the careful work of Dell and Elmedlaoui (2008). This means that they are systematically related, and we will propose that they are all the same meter at an abstract level (cf. Deo 2007). To make clear which properties are constant and which vary, we will use a maximally explicit way of presenting the meters. The expected

\footnotetext{
${ }^{18}$ L.LL.L never occurs line initially in independent meters, and neither does Ø.LL.L.

${ }^{19}$ It should be noted, however, that some of the longer meters occur with L.LL.L in positions where there is no counterpart with L.H.L. We will assume that this has to do with other factors than pure markedness (e.g. line length, text setting, or the like).
} 
finding is that meters should vary minimally, i.e. that the space circumscribed by the tradition will evolve around a core. Some meters will be closer to the core, others will be closer to the margin of the system as a whole, and we expect the system to be filled out by meters from the core and outwards, since that is what adherence to the important constraints for Tashlhiyt meter would predict. The notions of core and margin will be important when we evaluate gaps in the system, where missing meters closer to the core require an explanation (i.e. should preferably be systematic gaps rather than accidental ones), while missing meters on the margin are nonembarrassing.

Catalexis is marked with ' $\varnothing$ ' for each missing syllable, as in (24) above. The first table is organised in two parts according to whether the first verse foot has the heavy syllable in an even (E) or odd (O) position (Dell and Elmedlaoui 2008: 4, 38f.), and the number of verse feet. We shall use the E/O distinction as a convenient way to refer to whole meters via their first verse foot. The type-coding to the right will be used to keep track of meters in later groupings. ${ }^{20}$

Independent E meters

\begin{tabular}{|c|c|c|c|c|c|c|c|c|c|}
\hline $\mathrm{E} / \mathrm{O}$ & First & Second & Third & Fourth & Fifth & Sixth & Seventh & Fitted & Type \\
\hline E & L.H.L & LL.H & L.H .Ø & & & & & 3 & $1 \mathrm{~A}-\mathrm{b}$ \\
\hline $\mathrm{E}$ & L.H.L & LL.H & L.Ø .Ø & & & & & 3 & $1 \mathrm{~A}-\mathrm{c}$ \\
\hline E & L.H.L & LL.H & L.H .L & LL.H & & & & 4 & $2 \mathrm{~A}-\mathrm{a}$ \\
\hline $\mathrm{E}$ & L.H.L & LL.H & L.LL.L & LL.H & & & & 4 & $2 \mathrm{~B}-\mathrm{a}$ \\
\hline $\mathrm{E}$ & L.H.L & LL.H & L.H .L & LL. $\varnothing$ & & & & 4 & $2 \mathrm{~A}-\mathrm{b}$ \\
\hline $\mathrm{E}$ & L.H.L & LL.H & L.LL.L & LL. $\varnothing$ & & & & 4 & $2 \mathrm{~B}-\mathrm{b}$ \\
\hline $\mathrm{E}$ & L.H.L & LL.H & L.H .L & LØ.Ø & & & & 4 & $2 \mathrm{~A}-\mathrm{c}$ \\
\hline $\mathrm{E}$ & L.H.L & LL.H & L.LL.L & LØ.Ø & & & & 4 & $2 \mathrm{~B}-\mathrm{c}$ \\
\hline $\mathrm{E}$ & Ø.H.L & LL.H & L.H .L & LL.H & & & & 4 & $2 \mathrm{~A}-\mathrm{d}$ \\
\hline $\mathrm{E}$ & Ø.H.L & LL.H & L.LL.L & LL.H & & & & 4 & $2 B-d$ \\
\hline $\mathrm{E}$ & Ø.H.L & LL.H & L.H .L & LL. $\varnothing$ & & & & 4 & $2 \mathrm{~A}-\mathrm{e}$ \\
\hline $\mathrm{E}$ & Ø.H.L & LL.H & L.H .L & L $\varnothing . \varnothing$ & & & & 4 & $2 \mathrm{~A}-\mathrm{f}$ \\
\hline $\mathrm{E}$ & Ø.H.L & LL.H & L.H .L & LL.H & L. $\varnothing . \varnothing$ & & & 4 & $2 F A-i$ \\
\hline $\mathrm{E}$ & Ø.Ø.L & LL.H & L.H .L & LL.H & L.H.Ø & & & 4 & 2FA-ii \\
\hline $\mathrm{E}$ & $\varnothing . \varnothing . L$ & LL.H & L.LL.L & LL.H & L.H.Ø & & & 4 & 2FB-ii \\
\hline $\mathrm{E}$ & L.H.L & LL.H & L.H .L & LL.H & L.H.Ø & & & 5 & $3 \mathrm{~A}-\mathrm{b}$ \\
\hline $\mathrm{E}$ & L.H.L & LL.H & L.H .L & LL.H & L.Ø.Ø & & & 5 & $3 \mathrm{~A}-\mathrm{c}$ \\
\hline $\mathrm{E}$ & L.H.L & LL.H & L.H .L & LL.H & L.H.L & LL. $\varnothing$ & & 6 & $4 A-b$ \\
\hline $\mathrm{E}$ & L.H.L & LL.H & L.H .L & LL.H & L.H.L & L $\varnothing . \varnothing$ & & 6 & $4 \mathrm{~A}-\mathrm{c}$ \\
\hline
\end{tabular}

${ }^{20}$ The digit refers to the meter cluster. Uppercase letter A refers to LHL, B to LLLL, C to LLLL twice, lowercase a, b, c etc. to increasing catalexis, F to crucially fitted meters (see Sects. 5.3 and 5.5) and i-iii refers to variations in catalexis in crucially fitted meters. This notation is just for convenience since there are no class formations. 
(26) Independent $\mathrm{O}$ meters

\begin{tabular}{|c|c|c|c|c|c|c|c|c|c|}
\hline $\mathrm{E} / \mathrm{O}$ & First & Second & Third & Fourth & Fifth & Sixth & Seventh & Fitted & Type \\
\hline $\mathrm{O}$ & LL.H & L.LL.L & LL.H & & & & & 3 & $5 B-a$ \\
\hline $\mathrm{O}$ & LL.H & L.H .L & LL.H & L.H . . & & & & 4 & $6 \mathrm{~A}-\mathrm{b}$ \\
\hline $\mathrm{O}$ & LL.H & L.LL.L & LL.H & L.H .Ø & & & & 4 & $6 B-b$ \\
\hline $\mathrm{O}$ & LL.H & L.LL.L & LL.H & L.LL. $\varnothing$ & & & & 4 & $6 C-b$ \\
\hline $\mathrm{O}$ & LL.H & L.LL.L & LL.H & L.Ø .Ø & & & & 4 & $6 \mathrm{~B}-\mathrm{c}$ \\
\hline $\mathrm{O}$ & $\varnothing \mathrm{L} . \mathrm{H}$ & L.LL.L & LL.H & L.H .Ø & & & & 4 & $6 \mathrm{~B}-\mathrm{e}$ \\
\hline $\mathrm{O}$ & $\varnothing \mathrm{L} . \mathrm{H}$ & L.LL.L & LL.H & L.LL. $\varnothing$ & & & & 4 & $6 C-e$ \\
\hline $\mathrm{O}$ & $\varnothing \varnothing . H$ & L.LL.L & LL.H & L.H . . & & & & 4 & $6 B-g$ \\
\hline $\mathrm{O}$ & $\varnothing \varnothing . H$ & L.LL.L & LL.H & L.LL. $\varnothing$ & & & & 4 & $6 C-g$ \\
\hline $\mathrm{O}$ & $\varnothing \varnothing . H$ & L.H .L & LL.H & L.H .L & LØ.Ø & & & 4 & 6FA-iii \\
\hline $\mathrm{O}$ & $\varnothing \mathrm{L} . \mathrm{H}$ & L.LL.L & LL.H & L.H .L & LØ.Ø & & & 4 & $6 \mathrm{FB}-\mathrm{i}$ \\
\hline $\mathrm{O}$ & $\varnothing \mathrm{L} . \mathrm{H}$ & L.LL.L & LL.H & L.LL.L & LØ.Ø & & & 4 & $6 \mathrm{FC}-\mathrm{i}$ \\
\hline $\mathrm{O}$ & ØØ.Н & L.LL.L & LL.H & L.LL.L & LL.Ø & & & 4 & 6FC-ii \\
\hline $\mathrm{O}$ & $\varnothing \varnothing . Н$ & L.LL.L & LL.H & L.H .L & LØ.Ø & & & 4 & 6FB-iii \\
\hline $\mathrm{O}$ & $\varnothing \varnothing . H$ & L.LL.L & LL.H & L.LL.L & LØ.Ø & & & 4 & 6FC-iii \\
\hline $\mathrm{O}$ & ØØ.Н & L.LL.L & LL.H & L.LL.L & LL.H & L.H.L & LL. $\varnothing$ & 6 & 7FC-ii \\
\hline
\end{tabular}

\subsection{Complementarity and the fitted count}

Dell and Elmedlaoui (2008: 88ff.) provide extensive evidence for the fact that when different meters are combined in a stanza, this is done in such a manner that the longer sequences that are created are also straight or come close to being straight. Picking and combining meters at random would not yield that result, and so the combination is regulated. This regulation amounts to respecting verse foot alternation across meters and to the consideration of catalexis of abutting verse feet. They refer to this phenomenon as 'complementarity'. Combination of meters sometimes leaves a gap of one syllable, which may be filled by a so-called stop-gap vowel, or which may remain unfilled, i.e. catalectic. An example of full complementarity is given below (Dell and Elmedlaoui 2008: 89). The end of the first meter ([LL Ø) fits perfectly together with the beginning of the second meter $(\varnothing \mathrm{H}])$.

$$
\begin{array}{lll}
\text { Complementarity } & & \\
\text { [L.H.L] [LL } & \# & \text { H] [L.H.L] [LL. i] } \\
\text { a- yi ha wddi } & \# & \text { win ta-sas ti } \underline{\text { dh ru kan }} \\
\text { A yyih awddi } & \# & \text { win t-assas-t i-dhr=ukan } \\
\text { 'Oh yes, buddy' } & \# & \text { 'Everyone can see the poor wretch' }
\end{array}
$$

Something similar to complementarity appears to hold as a tendency also when you look at the beginning and end of the same meter. We will refer to this phenomenon as the 'fitted count' of verse feet, in anticipation of the discussion of preferred line length in Sect. 6.2. The fitted count sometimes differs from the 'structural count' of verse feet. These two measures count the number of verse feet in a meter either directly 
from one end to the other (structural), or by looking at how many verse feet a line would add up to if one pieced together its tail with its head (fitted). The difference between these modes of counting is illustrated in (28).

(28) Structural and fitted counts of verse feet

2FA-i

\begin{tabular}{|c|c|c|c|}
\hline \multirow{3}{*}{$\begin{array}{l}\text { Structural: } 5 \\
\text { Fitted: } 4\end{array}$} & [Ø.H.L] [LL.H] & [L.H.L] [LL.H] & [L.Ø.Ø] \\
\hline & .H.L] [LL.H] & [L.H.L] [LL.H] & {$[\mathrm{L}$.} \\
\hline & [LL.H] & [L.H.L] [LL.H] & [L.H.L] ) \\
\hline & 6FA-iii & & \\
\hline Structural: 5 & [ØØ.H] [L.H.L] & [LL.H] [L.H.L] & [LØ.Ø] \\
\hline Fitted: 4 & .H] [L.H.L] & [LL.H] [L.H.L] & [L $\varnothing$. \\
\hline (i.e. & [L.H.L] & [LL.H] [L.H.L] & [LØ.H] ) \\
\hline
\end{tabular}

Structural: 7 [ØØ.H] [L.LL.L] [LL.H] [L.LL.L] [LL.H] [L.H.L] [LL.Ø]

Fitted: 6 .H] [L.LL.L] [LL.H] [L.LL.L] [LL.H] [L.H.L] [LL.

(i.e. $\quad$ [L.LL.L] [LL.H] [L.LL.L] [LL.H] [L.H.L] [LL.H] ) 2A-e

Structural: 4 [Ø.H.L] [LL.H] [L.H.L] [LL.Ø]

Fitted: $4 \quad$ [Ø.H.L] [LL.H] [L.H.L] [LL. Ø]

(no difference)

A line of e.g. five verse feet under the structural count, which is catalectic at both ends may add up to four verse feet in the fitted count, e.g. 2FA-i. In the examples in (28), the fitted count could result in complete verse feet (2FA-i, 7FC-ii) or less (6FA-iii), as is also the case with complementarity. The fitted count only makes a difference for uneven structural counts (i.e. $7>6,5>4,3>2$ ), since only in uneven meters will the first and last verse foot be of the same type and therefore possible to fit together.

The fitted count reveals the binarity of the system more forcefully than the structural count. Most meters contain four verse feet. With four verse feet, a tetrameter, categories in Tashlhiyt Berber meter are binary all the way from the bottom (prosodic foot) to the top (meter). We return to binarity in Sect. 6.2 after all meters have been presented.

The rationale for fitted counting further resides in the fact that it reveals the similarity between meters in a way that the structural count doesn't. We return to this in Sect. 6.1.

\subsection{Descriptive groupings of independent meters}

There are no natural subgroupings within the straight meters. Instead, the metrical variation evolves in small steps around a prototypical centre. We shall describe this fact by dividing the independent and dependent meters into clusters based on (fitted) measure and surface resemblance. In a second step (Sect. 6.1), we will show how the 
boundaries between these clusters are illusory, the transitions between meters being seamless in all directions. This means that, even though it is practical to describe things in terms of clusters, there are ultimately no basic subtypes within the straight meters. In Sect. 6 we show instead that the metrical system of Tashlhiyt is of the same basic kind as the Sanskrit meters, as described by Deo (2007). That is to say there is a basic abstract meter, which is describable by a few desiderata, one of which is obligatory (clash avoidance), and some of which are emergent (binary lengths, lapse avoidance). The realizations of the abstract meter is what we refer to as the straight meters, i.e. as reified instantiations of the abstract meter, and whose patterns are repeated in individual poems. Dell and Elmedlaoui refer to them as MPs, i.e. metrical patterns.

\subsection{Clusters of independent meters}

We retain the convenient descriptive distinction of $\mathrm{O}$ meters (beginning in a LL.H verse foot) and E meters (beginning in a L.H.L verse foot) from Dell and Elmedlaoui, even though the $\mathrm{O} / \mathrm{E}$ distinction will ultimately prove to be spurious (Sect. 6). The independent meters can be grouped into seven clusters based on resemblance and measure (using the fitted count). These are presented in several tables below. In order to keep track of the shape variation between L.H.L and L.LL.L, we label meters A, B and C, where B and C indicate increasing use of L.LL.L instead of L.H.L. Catalectic verse feet of this kind are taken to be basically L.H.L, and will hence be labelled A. Distinctions based on catalexis are registered with lowercase letters $(\mathrm{a}-\mathrm{f})$, where a means acatalectic. The further into the alphabet, the more catalectic the meter. In presentations below, meters within a cluster are grouped according to the catalexis patterns (rather than verse foot shape variation). Meters where the structural and fitted counts amount to different numbers of verse feet are marked with an F (=crucially fitted). These meters are obviously richly catalectic and their catalexis profile is marked with roman numbers (i-iii). A number of absent but hypothetical meters are included. These are set in italics and are prefixed with a star '*', which is also registered in a column to the right, followed by an indication of the property that we assume causes the exclusion of the meter from the set of attested straight meters. Hypothetical meters that are included mostly constitute systematic or accidental gaps, whereas meters that belong on the margin are given as examples, but are not exhaustively registered. We begin with the E meters.

Cluster 1, three fitted verse feet (E)

\begin{tabular}{lllllll}
\hline & Type & First & Second & Third & & \\
\hline $\mathrm{E}$ & $* 1 \mathrm{~A}-\mathrm{a}$ & L.H.L & LL.H & L.H.L & $*$ & endlapse \\
$\mathrm{E}$ & $1 \mathrm{~A}-\mathrm{b}$ & L.H.L & LL.H & L.H.Ø & & \\
$\mathrm{E}$ & 1A-c & L.H.L & LL.H & L.Ø.Ø & & \\
\hline
\end{tabular}


In this cluster there are two short independent meters, both of them final catalectic. ${ }^{21}$ This leads us to look for a reason why the acatalectic meter is missing (*1A-a). The acatalectic shape of any meter is less marked than catalectic shapes, and so the absence of acatalectic meters should hardly be accidental gaps. In this case, and in several corresponding ones below, the gap is systematic and due to the lapse in the final verse foot (marked 'endlapse', see (22)). Also, there is no variation between L.H.L and L.LL.L in this family, making the cluster small. As mentioned, there is a general exclusion of L.LL.L and catalectic variants thereof in the line-initial position, cf. (24). Meters that are shorter than those in (29) are dependent (Sect. 5.6), and longer meters belong in cluster 2 given in (30).

(30) Cluster 2, four fitted verse feet $(\mathrm{E})$

\begin{tabular}{|c|c|c|c|c|c|c|c|c|}
\hline & Type & First & Second & Third & Fourth & Fifth & & \\
\hline $\mathrm{E}$ & $2 \mathrm{~A}-\mathrm{a}$ & L.H.L & LL.H & L.H .L & LL.H & & & \\
\hline $\mathrm{E}$ & $2 \mathrm{~B}-\mathrm{a}$ & L.H.L & LL.H & L.LL.L & LL.H & & & \\
\hline $\mathrm{E}$ & $2 \mathrm{~A}-\mathrm{b}$ & L.H.L & LL.H & L.H .L & LL. $\varnothing$ & & & \\
\hline $\mathrm{E}$ & $2 \mathrm{~B}-\mathrm{b}$ & L.H.L & LL.H & L.LL.L & LL. $\varnothing$ & & & \\
\hline $\mathrm{E}$ & $2 \mathrm{~A}-\mathrm{c}$ & L.H.L & LL.H & L.H .L & LØ.Ø & & & \\
\hline $\mathrm{E}$ & $2 B-c$ & L.H.L & LL.H & L.LL.L & LØ.Ø & & & \\
\hline $\mathrm{E}$ & $2 \mathrm{~A}-\mathrm{d}$ & Ø.H.L & LL.H & L.H .L & LL.H & & & \\
\hline $\mathrm{E}$ & $2 B-d$ & Ø.H.L & LL.H & L.LL.L & LL.H & & & \\
\hline $\mathrm{E}$ & $2 \mathrm{~A}-\mathrm{e}$ & Ø.H.L & LL.H & L.H .L & LL. $\varnothing$ & & & \\
\hline $\mathrm{E}$ & $* 2 \mathrm{~B}-\mathrm{e}$ & Ø.H.L & $L L . H$ & L.LL.L & $L L . \varnothing$ & & $*$ & margin \\
\hline $\mathrm{E}$ & $2 \mathrm{~A}-\mathrm{f}$ & Ø.H.L & LL.H & L.H .L & L $\varnothing . \varnothing$ & & & \\
\hline $\mathrm{E}$ & $* 2 \mathrm{~B}-\mathrm{f}$ & Ø.H.L & LL.H & L.LL.L & $L \varnothing . \varnothing$ & & $*$ & margin \\
\hline $\mathrm{E}$ & $2 F A-i$ & Ø.H.L & LL.H & L.H .L & LL.H & L.Ø.Ø & & \\
\hline $\mathrm{E}$ & 2FA-ii & $\varnothing . \varnothing . L$ & LL.H & L.H .L & LL.H & L.H.Ø & & \\
\hline $\mathrm{E}$ & 2FB-ii & Ø.Ø.L & LL.H & L.LL.L & LL.H & L.H.Ø & & \\
\hline
\end{tabular}

Cluster 2 is large, exhibiting variation in the shape of the third anaclastic verse foot, and catalexis at both ends. The meter of ndalb irbbi is included here (2B-a). The fitted count is crucial in the last three meters, which contain four crucially fitted (five structural) verse feet. Two missing meters have been included. These are simply variations in the third verse foot, and there is no particular reason why they should be missing. These are potential meters, but located on the margin of the metrical space employed by the straight meters (therefore marked 'margin', cf. Sect. 5.2). Their presence or absence cannot be predicted or attributed to some systematic property.

${ }^{21} 1 \mathrm{~A}-\mathrm{c}$ is the meter of the refrain of Ndalb irbbi in (11). 
In particular, if L.H.L is taken as more basic than L.LL.L, these would be a priori less expected than their corresponding, extant, neighbouring meters (2A-e and $2 \mathrm{~A}-\mathrm{f}){ }^{22}$

Cluster 3, five fitted verse feet (E)

\begin{tabular}{|c|c|c|c|c|c|c|c|c|}
\hline & Type & First & Second & Third & Fourth & Fifth & & \\
\hline $\mathrm{E}$ & $* 3 \mathrm{~A}-\mathrm{a}$ & L.H.L & LL.H & L.H.L & LL.H & L.H.L & 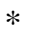 & endlapse \\
\hline $\mathrm{E}$ & $3 A-b$ & L.H.L & LL.H & L.H.L & LL.H & L.H.Ø & & \\
\hline $\mathrm{E}$ & $3 \mathrm{~A}-\mathrm{c}$ & L.H.L & LL.H & L.H.L & LL.H & L.Ø.Ø & & \\
\hline
\end{tabular}

As in cluster 1, both meters in cluster 3 are final catalectic, and the acatalectic meter is missing, for a reason of principle (endlapse, (22)). There is no variation between L.H.L and L.LL.L neither here nor in cluster 4.

Cluster 4, six fitted verse feet $(\mathrm{E})$

\begin{tabular}{llllllllll}
\hline & Type & First & Second & Third & Fourth & Fifth & \multicolumn{2}{l}{ Sixth } & \\
\hline E & $* 4$ A-a & L.H.L & LL.H & L.H.L & LL.H & L.H.L & LL.H & $*$ & gap \\
E & 4A-b & L.H.L & LL.H & L.H.L & LL.H & L.H.L & LL. & & \\
E & 4A-c & L.H.L & LL.H & L.H.L & LL.H & L.H.L & LØ. & & \\
\hline
\end{tabular}

This cluster is again small, containing only two meters, both final catalectic. The acatalectic meter is missing and we have no explanation for this. Rather it appears to be a real accidental gap (marked 'gap'). We now turn to the O clusters.

Cluster 5, three fitted verse feet $(\mathrm{O})$

\begin{tabular}{lllllll}
\hline & Type & First & Second & Third & \\
\hline $\mathrm{O}$ & $* 5 \mathrm{~A}-\mathrm{a}$ & LL.H & L.H.L & LL.H & $*$ & gap \\
$\mathrm{O}$ & $5 \mathrm{~B}-\mathrm{a}$ & LL.H & L.LL.L & LL.H & & \\
\hline
\end{tabular}

Cluster 5 contains only one short acatalectic meter. The missing meter provided is the one with a variant shape in the second verse foot. If L.H.L is more basic than L.LL.L, this is slightly unexpected. The smallness of this cluster is actually an artefact of the description. Once we look at the dependent meters we shall see that this meter is simply at the boundary between the independent and dependent meters (Sect. 5.6).

\footnotetext{
${ }^{22}$ The meter called $2 \mathrm{~A}-\mathrm{f}$ could in principle be given a fitted count with three verse feet, but that would mean the fitting of structurally different verse feet, which we do not otherwise find within the independent meters.
} 
(34) Cluster 6, four fitted verse feet $(\mathrm{O})$

\begin{tabular}{|c|c|c|c|c|c|c|c|c|}
\hline & Type & First & Second & Third & Fourth & Fifth & & \\
\hline & $* 6 \mathrm{~A}-\mathrm{a}$ & LL.H & $L . H . L$ & LL.H & $L . H \quad . L$ & & $*$ & endlapse \\
\hline $\mathrm{O}$ & $* 6 \mathrm{~B}-\mathrm{a}$ & LL.H & L.LL.L & LL.H & $L . H \quad . L$ & & $*$ & endlapse \\
\hline $\mathrm{O}$ & $* 6 \mathrm{C}-\mathrm{a}$ & LL.H & L.LL.L & LL.H & L.LL.L & & $*$ & endlapse \\
\hline $\mathrm{O}$ & $6 A-b$ & LL.H & L.H .L & LL.H & L.H .Ø & & & \\
\hline $\mathrm{O}$ & $6 B-b$ & LL.H & L.LL.L & LL.H & L.H .Ø & & & \\
\hline $\mathrm{O}$ & $6 C-b$ & LL.H & L.LL.L & LL.H & L.LL. $\varnothing$ & & & \\
\hline $\mathrm{O}$ & $6 \mathrm{~B}-\mathrm{c}$ & LL.H & L.LL.L & LL.H & L.Ø .Ø & & & \\
\hline $\mathrm{O}$ & $* 6 \mathrm{~A}-\mathrm{d}$ & $\emptyset L . H$ & L.H .L & LL.H & $L . H \quad . L$ & & $*$ & endlapse \\
\hline $\mathrm{O}$ & $* 6 \mathrm{~B}-\mathrm{d}$ & $\varnothing L . H$ & L.LL.L & LL.H & L.H .L & & $*$ & endlapse \\
\hline $\mathrm{O}$ & $* 6 \mathrm{C}-\mathrm{d}$ & $\varnothing L . H$ & L.LL.L & LL.H & $L . L L . L$ & & $*$ & endlapse \\
\hline $\mathrm{O}$ & $6 \mathrm{~B}-\mathrm{e}$ & $\varnothing \mathrm{L} . \mathrm{H}$ & L.LL.L & LL.H & L.H .Ø & & & \\
\hline $\mathrm{O}$ & 6C-e & $\varnothing \mathrm{L} . \mathrm{H}$ & L.LL.L & LL.H & L.LL. $\varnothing$ & & & \\
\hline $\mathrm{O}$ & $* 6 \mathrm{~B}-\mathrm{f}$ & $\varnothing L . H$ & L.LL.L & LL.H & $L . \varnothing . \varnothing$ & & $*$ & margin \\
\hline $\mathrm{O}$ & $6 \mathrm{~B}-\mathrm{g}$ & $\varnothing \varnothing . H$ & L.LL.L & LL.H & L.H .Ø & & & \\
\hline $\mathrm{O}$ & $6 C-g$ & $\varnothing \varnothing . H$ & L.LL.L & LL.H & L.LL. $\varnothing$ & & & \\
\hline $\mathrm{O}$ & 6FA-iii & $\varnothing \varnothing . H$ & L.H .L & LL.H & L.H .L & L $\varnothing . \varnothing$ & & \\
\hline $\mathrm{O}$ & $* 6 \mathrm{FA}-\mathrm{i}$ & $\varnothing L . H$ & L.LL.L & LL.H & $L . H \quad . L$ & $L \emptyset . \varnothing$ & $*$ & margin \\
\hline $\mathrm{O}$ & $6 \mathrm{FB}-\mathrm{i}$ & $\varnothing \mathrm{L} . \mathrm{H}$ & L.LL.L & LL.H & L.H .L & LØ.Ø & & \\
\hline $\mathrm{O}$ & $6 \mathrm{FC}-\mathrm{i}$ & $\varnothing \mathrm{L} . \mathrm{H}$ & L.LL.L & LL.H & L.LL.L & LØ.Ø & & \\
\hline $\mathrm{O}$ & $* 6 \mathrm{FA}-\mathrm{ii}$ & $\varnothing \varnothing . H$ & L.H .L & LL.H & $L . H \quad . L$ & LL. $\varnothing$ & $*$ & margin \\
\hline $\mathrm{O}$ & $* 6 \mathrm{FB}-\mathrm{ii}$ & $\varnothing \varnothing . H$ & L.LL.L & LL.H & $L . H \quad . L$ & $L L . \varnothing$ & $*$ & margin \\
\hline $\mathrm{O}$ & 6FC-ii & $\varnothing \varnothing . H$ & L.LL.L & LL.H & L.LL.L & LL. $\varnothing$ & & \\
\hline $\mathrm{O}$ & 6FA-iii & $\varnothing \varnothing . H$ & $L . H \quad . L$ & LL.H & $L . H \quad . L$ & $L \emptyset . \emptyset$ & $*$ & margin \\
\hline $\mathrm{O}$ & 6FB-iii & $\varnothing \varnothing . H$ & L.LL.L & LL.H & L.H .L & LØ.Ø & & \\
\hline $\mathrm{O}$ & 6FC-iii & $\varnothing \varnothing . H$ & L.LL.L & LL.H & L.LL.L & LØ.Ø & & \\
\hline
\end{tabular}

Cluster 6 is the largest of all clusters, containing fourteen meters, several of them crucially fitted in the verse foot count. The first two groups of missing meters are acatalectic at the right end, again due to endlapse (22) which causes several systematic gaps in this system.

The rest of the missing meters are all on the margin of the metrical space, i.e. these are meters that are predicted to be well-formed members of the cluster, but which are close to the outer boundaries of the metrical system, hence have not (yet) been recruited (or collected). We may note that there is only one A-meter in this group (containing L.H.L in all possible positions). For some reason, the first of the anaclastic verse feet in this meter is preferably L.LL.L, rather than L.H.L.

Cluster 7, six fitted verse feet $(\mathrm{O})$

Type First Second Third Fourth Fifth Sixth Seventh

O *7C-a LL.H L.LL.L LL.H L.LL.L LL.H L.H.L LL.H * length

O 7FC-ii $\varnothing . H$ L.LL.L LL.H L.LL.L LL.H L.H.L LL. $\varnothing$ 
The last group, cluster 7, contains only one meter and it has a crucially fitted count of verse feet, which brings the number of verse feet down to six. This would seem to be the longest attested meter in this system. If so, it accounts for the absence of less catalectic or acatalectic meters in this cluster (and beyond), since they would all be too long. No meter in the system has seven or more verse feet in the fitted count. We register this hypothesis as 'length' to the right, and we assume that this is one of the margins of the system.

Catalectic verse feet occur both finally and initially in lines, never inside lines. Cross-linguistically, final catalexis is more common than initial catalexis and Tashlhiyt Berber is no exception. While several meter clusters have both final and initial catalexis, no cluster has initial catalexis to the exclusion of final catalexis. But three clusters have only final catalexis (1, 3 and 4).

\subsection{Dependent meters}

The 21 dependent meters make up five clusters (8-12) based on measure. Many of their properties are like the independent meters, but they are all short, apparently the property that makes them dependent. They vary between one and three verse feet in length, with a strong preponderance for two verse feet (17 out of 21 , cf. (46)). Unlike the independent meters, the dependent meters may begin with the L.LL.L verse foot. Also there are two dependent meters which end in a lapsing verse foot $(8 \mathrm{~B}$ and $10 \mathrm{~A}-\mathrm{d})$, though not freely $(* 11 \mathrm{~A}-\mathrm{a}$ and $* 11 \mathrm{~B}-\mathrm{a}){ }^{23} \mathrm{We}$ present the dependent meters in two tables. A few accidental gaps are registered where the anaclastic verse foot L.H.L is missing and L.LL.L is attested in the corpus.

(36) Dependent E meters, clusters 8-10

\begin{tabular}{|c|c|c|c|c|c|c|}
\hline & Type & First & Second & Third & Fitted & \\
\hline $\mathrm{E}$ & $* 8 \mathrm{~A}$ & L.H .L & & & $1 *$ & endlapse dependent, gap \\
\hline $\mathrm{E}$ & $8 \mathrm{~B}$ & L.LL.L & & & 1 & endlapse dependent \\
\hline $\mathrm{E}$ & $9 \mathrm{~A}-\mathrm{a}$ & L.H .L & LL.H & & 2 & \\
\hline $\mathrm{E}$ & $9 A-b$ & L.H .L & LL. $\varnothing$ & & 2 & \\
\hline $\mathrm{E}$ & $9 B-b$ & L.LL.L & LL. $\varnothing$ & & 2 & \\
\hline $\mathrm{E}$ & $9 \mathrm{~A}-\mathrm{c}$ & L.H .L & LØ.Ø & & 2 & \\
\hline $\mathrm{E}$ & 9B-c & L.LL.L & LØ.Ø & & 2 & \\
\hline $\mathrm{E}$ & 9FA-i & $\varnothing . \mathrm{H} . \mathrm{L}$ & LL.H & L. $\varnothing . \varnothing$ & 2 & \\
\hline $\mathrm{E}$ & 9FB-ii & $\varnothing . \varnothing . \mathrm{L}$ & LL.H & L.LL.O & 2 & \\
\hline $\mathrm{E}$ & 9FA-iii & $\varnothing . \varnothing . \mathrm{L}$ & LL.H & L. $\varnothing . \varnothing$ & 2 & \\
\hline $\mathrm{E}$ & 10A-d & $\emptyset . \mathrm{H} . \mathrm{L}$ & LL.H & L.H .L & 3 & endlapse dependent \\
\hline $\mathrm{E}$ & $10 \mathrm{~A}-\mathrm{e}$ & Ø.H .L & LL.H & L.H .Ø & 3 & \\
\hline
\end{tabular}

${ }^{23}$ We would hypothesize that these meters combine with preceding and following independent meters in such a way that stanza-final lapse and line-initial L.LL.L are avoided. The workings of NOLAPSE (avoiding endlapse) apply in stanzas rather than in lines of meter. 
Dependent O meters, clusters 11-12

\begin{tabular}{|c|c|c|c|c|c|c|}
\hline & Type & First & Second & Third & Fitted & \\
\hline $\mathrm{O}$ & $* 11 \mathrm{~A}-\mathrm{a}$ & LL.H & $L . H \quad . L$ & & $2 *$ & endlapse dependent \\
\hline $\mathrm{O}$ & $* 11 \mathrm{~B}-\mathrm{a}$ & LL.H & L.LL.L & & $2 *$ & endlapse dependent \\
\hline $\mathrm{O}$ & $* 11 \mathrm{~A}-\mathrm{b}$ & LL.H & L.H. .Ø & & $2 *$ & gap \\
\hline $\mathrm{O}$ & 11B-b & LL.H & L.LL. $\varnothing$ & & 2 & \\
\hline $\mathrm{O}$ & $11 \mathrm{~A}-\mathrm{c}$ & LL.H & L.Ø .Ø & & 2 & \\
\hline $\mathrm{O}$ & $11 \mathrm{~A}-\mathrm{d}$ & $\varnothing \mathrm{L} . \mathrm{H}$ & L.H .Ø & & 2 & \\
\hline $\mathrm{O}$ & 11B-d & $\varnothing \mathrm{L} . \mathrm{H}$ & L.LL. $\varnothing$ & & 2 & \\
\hline $\mathrm{O}$ & *11FA-i & $\varnothing L . H$ & $L . H \quad . L$ & $L \emptyset . \emptyset$ & $2 *$ & gap \\
\hline $\mathrm{O}$ & 11FB-i & $\varnothing \mathrm{L} . \mathrm{H}$ & L.LL.L & L $\varnothing . \varnothing$ & 2 & \\
\hline $\mathrm{O}$ & 11FA-iii & $\varnothing \varnothing . H$ & L.H .L & L $\varnothing . \varnothing$ & 2 & \\
\hline $\mathrm{O}$ & 11FB-iii & $\varnothing \varnothing . H$ & L.LL.L & LØ.Ø & 2 & \\
\hline $\mathrm{O}$ & 11FA-ii & $\varnothing \varnothing . H$ & L.H .L & LL. $\varnothing$ & 2 & \\
\hline $\mathrm{O}$ & 11FB-ii & $\varnothing \varnothing . H$ & L.LL.L & LL. $\varnothing$ & 2 & \\
\hline $\mathrm{O}$ & $12 \mathrm{~A}-\mathrm{x}$ & $\varnothing \varnothing . H$ & L.H .L & LL.H & 3 & \\
\hline
\end{tabular}

The last meter here has the property of two initial catalectic syllables combined with an acatalectic ending, a property not found in the independent meters. Whether this has any implications for the way it combines with other meters cannot be established without looking at more data than what is available to us.

\section{A continuous metrical space}

As stated in Sect. 5.4, the categorization into clusters is superficial, as the straight meters appear to circumscribe a continuous metrical space by minimal variation in several dimensions. The aim now is to show how the meters form a continuum. To do this we focus on the transitions between the descriptive categories that we have introduced.

The upshot is that the straight meters of Tashlhiyt Berber form a single metrical family, in the same sense as groups of meters in classical Sanskrit form metrical families (Deo 2007). Deo's insight was that the large number of meters in Sanskrit could be reduced to just a few, more abstract metrical schemata. The instantiation of meters (which carry names in the long history of Sanskrit metrical analysis and nomenclature) as single, fixed realizations of those metrical schemata, are then repeated throughout a single poem (typically with no variable positions). In the classical western tradition this is known as responsion (Swift 2013), where it usually refers to the requirement that some line towards the end of a poem be metrically identical to some line in the beginning of that poem, a limited phenomenon. Sanskrit meter is fully responding, i.e. responsion is generalized throughout a poem, such that every line must be metrically identical to the preceding one. ${ }^{24}$

The Tashlhiyt Berber straight meters form a family in the same sense. Every line is identical to the preceding one regarding length, distribution of verse feet and the

${ }^{24}$ Deo (2007) shows that this is not fully true for all metrical families, including the Indravajrā. 
shape of verse feet (L.H.L/L.LL.L). However, Tashlhiyt goes beyond what's reported for Sanskrit, in that there is no obvious sense in which the length of the Tashlhiyt straight meters is fixed at the level of the abstract metrical schema. Thus, where (the analysis of) Sanskrit has separate metrical schemata for (trochaic, iambic or mixed) tetrameter and pentameter, the corresponding lengths in Tashlhiyt are not obviously separate. This is due to the fine-grained modulation of catalexis, creating a continuum of line lengths and verse foot alternation. The notions of complementarity and fitted count are part of this continuous modulation. We will illustrate these points by looking at transitions between the descriptive categories introduced above and showing that they are non-categorical.

\subsection{Transitions between types and clusters}

The presentation of meters above was primarily designed to give an overview of the system of meter clusters in terms of their number of fitted verse feet. However, patterns pertaining to the system as a whole have not yet been fully revealed and in this section we organize the same data differently in order to bring out further generalizations, all serving to make explicit the point that the meters form a coherent set with very few internal accidental gaps. One thing to demonstrate is that there is no firewall between the dependent and independent meters. The boundary between them is simply a matter of length, which may ultimately be due to the musical structure of the tradition.

The first table below includes E meters only, i.e. meters beginning with L.H.L or L.LL.L. In the head of the table we simply list a generic meter of alternating verse foot types. The variable ' $\varphi$ ' for $\{H, L L\}$ covers the middle position of the class of lapsing verse feet, L.H.L and L.LL.L. Status as independent or dependent is registered in the rightmost column (as well as in the type-coding to the left). The table in (38) is made up of all the dependent E-meters (see (36)) and the independent meters of cluster 1 (see (29)).

Dependent to independent and back to dependent

\begin{tabular}{|c|c|c|c|c|c|c|}
\hline & Type & L. $\varphi . L$ & LL.H & L. $\varphi . \mathrm{L}$ & & \\
\hline $\mathrm{E}$ & 8 & L.LL.L & & & & endlapse dependent \\
\hline $\mathrm{E}$ & $9 B-c$ & L.LL.L & L $\varnothing . \varnothing$ & & & dependent \\
\hline $\mathrm{E}$ & $9 \mathrm{~A}-\mathrm{c}$ & L.H .L & LØ.Ø & & & dependent \\
\hline $\mathrm{E}$ & $9 A-b$ & L.H .L & LL. $\varnothing$ & & & dependent \\
\hline $\mathrm{E}$ & $9 B-b$ & L.LL.L & LL. $\varnothing$ & & & dependent \\
\hline $\mathrm{E}$ & $9 \mathrm{~A}-\mathrm{a}$ & L.H .L & LL.H & & & dependent \\
\hline $\mathrm{E}$ & $1 \mathrm{~A}-\mathrm{c}$ & L.H .L & LL.H & L. $\varnothing . \varnothing$ & & independent \\
\hline $\mathrm{E}$ & $1 \mathrm{~A}-\mathrm{b}$ & L.H .L & LL.H & L.H ..$\varnothing$ & & independent \\
\hline $\mathrm{E}$ & $* 1 \mathrm{~A}-\mathrm{a}$ & $L . H \quad . L$ & LL.H & $L . H \quad . L$ & $*$ & endlapse independent \\
\hline $\mathrm{E}$ & $10 \mathrm{~A}-\mathrm{d}$ & $\varnothing . \mathrm{H} . \mathrm{L}$ & LL.H & L.H .L & & endlapse dependent \\
\hline $\mathrm{E}$ & $10 \mathrm{~A}-\mathrm{e}$ & Ø.H .L & LL.H & L.H .. & & dependent \\
\hline $\mathrm{E}$ & 9FA-i & Ø.H .L & LL.H & L. $\varnothing . \varnothing$ & & dependent \\
\hline $\mathrm{E}$ & 9FB-ii & $\varnothing . \varnothing . \mathrm{L}$ & LL.H & L.LL. $\varnothing$ & & dependent \\
\hline $\mathrm{E}$ & 9FA-iii & $\varnothing . \varnothing . \mathrm{L}$ & LL.H & L. $\varnothing . \varnothing$ & & dependent \\
\hline
\end{tabular}


We have put the missing, endlapsing ternary meter in the middle of this table, as a structural reference point. ${ }^{25}$ As a potential independent meter, it is excluded by NoLAPSE. Directly above it are two independent meters, followed upwards by dependent meters. Below the reference meter, all meters are dependent. The first dependent meter has an endlapse and it is predicted that this meter should precede whatever meter it combines with. We see clearly that the transition from dependent to independent and back to dependent is seamless, testifying to the fact that the system is modified in minimal steps, leaving the distinction between dependent and independent as simply a matter of length and structure. There is no clean cut-off point between independent and dependent meters, and, in terms of the number of metrical positions, the independent $1 \mathrm{~A}-\mathrm{b}$ and $1 \mathrm{~A}-\mathrm{c}$ are as long as the dependent $10 \mathrm{~A}-\mathrm{d}$ and $10 \mathrm{~A}-\mathrm{e}$.

Table (39) illustrates the same pattern with O meters, beginning with a LL.H verse foot. Here we have collected independent meters that border on closely related dependent meters. This table includes all dependent meters given in (37) and a number of independent meters.

Independent to dependent

\begin{tabular}{|c|c|c|c|c|c|c|c|}
\hline & Type & LL.H & L. $\varphi . \mathrm{L}$ & LL.H & L. $\varphi . L$ & & \\
\hline $\mathrm{O}$ & $6 B-g$ & $\varnothing \varnothing . H$ & L.LL.L & LL.H & L.H .Ø & & independent \\
\hline $\mathrm{O}$ & $6 \mathrm{C}-\mathrm{g}$ & $\varnothing \varnothing . H$ & L.LL.L & LL.H & L.LL. $\varnothing$ & & independent \\
\hline $\mathrm{O}$ & $6 \mathrm{~B}-\mathrm{e}$ & $\varnothing \mathrm{L} . \mathrm{H}$ & L.LL.L & LL.H & L.H .Ø & & ndent \\
\hline $\mathrm{O}$ & $6 C-e$ & $\varnothing \mathrm{L} . \mathrm{H}$ & L.LL.L & LL.H & L.LL. $\varnothing$ & & independent \\
\hline $\mathrm{O}$ & $* 6 \mathrm{~A}-\mathrm{a}$ & $L L . H$ & L.H .L & $L L . H$ & L.H .L & $*$ & endlapse \\
\hline $\mathrm{O}$ & $6 \mathrm{~A}-\mathrm{b}$ & LL.H & L.H .L & LL.H & L.H .Ø & & independent \\
\hline $\mathrm{O}$ & $6 \mathrm{~B}-\mathrm{b}$ & LL.H & L.LL.L & LL.H & L.H .Ø & & independent \\
\hline $\mathrm{O}$ & $6 C-b$ & LL.H & L.LL.L & LL.H & L.LL. $\varnothing$ & & Ident \\
\hline $\mathrm{O}$ & $6 \mathrm{~B}-\mathrm{c}$ & LL.H & L.LL.L & LL.H & L. $\varnothing . \varnothing$ & & independent \\
\hline $\mathrm{O}$ & $5 \mathrm{~B}-\mathrm{a}$ & LL.H & L.LL.L & LL.H & & & independent \\
\hline $\mathrm{O}$ & $* 5 B-d$ & $\varnothing L . H$ & $L . L L . L$ & $L L . H$ & & $*$ & gap \\
\hline $\mathrm{O}$ & $12 \mathrm{~A}-\mathrm{x}$ & $\varnothing \varnothing . H$ & L.H .L & LL.H & & & dependent \\
\hline $\mathrm{O}$ & 11FA-ii & ØØ.Н & L.H .L & LL.Ø & & & dependent \\
\hline $\mathrm{O}$ & 11FB-ii & $\varnothing \varnothing . H$ & L.LL.L & LL.Ø & & & dependent \\
\hline $\mathrm{O}$ & 11FB-i & $\varnothing \mathrm{L} . \mathrm{H}$ & L.LL.L & LØ.Ø & & & dependent \\
\hline $\mathrm{O}$ & 11FA-iii & $\varnothing \varnothing . H$ & L.H .L & LØ.Ø & & & dependent \\
\hline $\mathrm{O}$ & 11FB-iii & $\varnothing \varnothing . H$ & L.LL.L & LØ.Ø & & & dependent \\
\hline $\mathrm{O}$ & $* 11 \mathrm{~A}-\mathrm{a}$ & $L L . H$ & L.H .L & & & $*$ & endlapse \\
\hline $\mathrm{O}$ & $* 11 \mathrm{~B}-\mathrm{a}$ & $L L . H$ & L.LL.L & & & $*$ & endlapse \\
\hline $\mathrm{O}$ & $11 \mathrm{~B}-\mathrm{b}$ & LL.H & L.LL. $\varnothing$ & & & & dependent \\
\hline $\mathrm{O}$ & $11 \mathrm{~A}-\mathrm{d}$ & $\varnothing \mathrm{L} . \mathrm{H}$ & L.H .Ø & & & & dependent \\
\hline $\mathrm{O}$ & $11 \mathrm{~B}-\mathrm{d}$ & $\varnothing \mathrm{L} . \mathrm{H}$ & L.LL. $\varnothing$ & & & & dependent \\
\hline $\mathrm{O}$ & $11 \mathrm{~A}-\mathrm{c}$ & LL.H & L. $\varnothing . \varnothing$ & & & & dependent \\
\hline
\end{tabular}

\footnotetext{
${ }^{25}$ Reference points like this will be supplied in several tables below. They are chosen to highlight the pattern demonstrated in each table. In this case, ${ }^{*} 1 \mathrm{~A}-\mathrm{a}$ is the structural neighbour of both $1 \mathrm{~A}-\mathrm{b}$ and $10 \mathrm{~A}-\mathrm{d}$.
} 
This table is organized with independent meters at the top and the dependent meters below. The acatalectic tetrameter *6A-a is missing because of its endlapsing verse foot. Below $* 6 \mathrm{~A}$-a, final catalexis successively reduces the meters down to the ternary independent meter 5B-a. We then have a missing meter, *5B-d, presumably an accidental gap. All meters below are dependent and increasingly shorter by catalexis at either end or both. ${ }^{26}$ Tables (38) and (39) include all dependent meters given in Dell and Elmedlaoui (2008).

The table in (40) starts and ends with the same trimeter, used here as reference point for gradually expanding and shrinking tetrameters, with the acatalectic tetrameters in the centre (2A-a, 2B-a). This table contains all the attested meters of cluster 2 (see (30)) which are not crucially fitted.

(40) Independent trimeter to tetrameter and back

\begin{tabular}{|c|c|c|c|c|c|c|c|}
\hline & Type & L. $\varphi . \mathrm{L}$ & LL.H & L. $\varphi . \mathrm{L}$ & LL.H & & \\
\hline $\mathrm{E}$ & $1 \mathrm{~A}-\mathrm{b}$ & L.H.L & LL.H & L.H .Ø & & & independent \\
\hline $\mathrm{E}$ & $* 1 \mathrm{~A}-\mathrm{a}$ & L.H.L & $L L . H$ & L.H .L & & $*$ & endlapse independent \\
\hline $\mathrm{E}$ & $2 \mathrm{~A}-\mathrm{c}$ & L.H.L & LL.H & L.H .L & LØ.Ø & & independent \\
\hline $\mathrm{E}$ & $2 \mathrm{~B}-\mathrm{c}$ & L.H.L & LL.H & L.LL.L & LØ.Ø & & independent \\
\hline $\mathrm{E}$ & $2 \mathrm{~A}-\mathrm{b}$ & L.H.L & LL.H & L.H .L & LL. $\varnothing$ & & independent \\
\hline $\mathrm{E}$ & $2 \mathrm{~B}-\mathrm{b}$ & L.H.L & LL.H & L.LL.L & LL. $\varnothing$ & & independent \\
\hline $\mathrm{E}$ & $2 \mathrm{~A}-\mathrm{a}$ & L.H.L & LL.H & L.H .L & LL.H & & independent \\
\hline $\mathrm{E}$ & $2 \mathrm{~B}-\mathrm{a}$ & L.H.L & LL.H & L.LL.L & LL.H & & independent \\
\hline $\mathrm{E}$ & $2 \mathrm{~A}-\mathrm{d}$ & Ø.H.L & LL.H & L.H .L & LL.H & & independent \\
\hline $\mathrm{E}$ & $2 \mathrm{~B}-\mathrm{d}$ & $\varnothing . H . L$ & LL.H & L.LL.L & LL.H & & independent \\
\hline $\mathrm{E}$ & $2 \mathrm{~A}-\mathrm{e}$ & $\varnothing . H . L$ & LL.H & L.H .L & LL. $\varnothing$ & & independent \\
\hline $\mathrm{E}$ & $2 \mathrm{~A}-\mathrm{f}$ & $\varnothing . H . L$ & LL.H & L.H .L & LØ.Ø & & independent \\
\hline $\mathrm{E}$ & $* 1 \mathrm{~A}-\mathrm{a}$ & L.H.L & $L L . H$ & L.H .L & & $*$ & endlapse independent \\
\hline $\mathrm{E}$ & $1 \mathrm{~A}-\mathrm{b}$ & L.H.L & LL.H & L.H .. & & & independent \\
\hline
\end{tabular}

All initial catalectic, closely related meters are given in the lower half of the table. The chart in (38) could be seamlessly attached to the chart in (40). This could be done by splitting (38) at the reference meter *1A-a and adding the top half above and the bottom half below the meters in (40), or by conceiving of the meters as relating to each other in a three dimensional space (necessary if all straight meters were to be represented in a single chart).

The next table starts from the meters $2 \mathrm{~A}-\mathrm{a}$ and $2 \mathrm{~B}-\mathrm{a}$ of item (40) (only $2 \mathrm{~A}-\mathrm{a}$ is repeated here), and shows the successive expansion of meters mostly to the right but also to the left in the last extant meter (7FC-ii).

\footnotetext{
${ }^{26}$ We cannot tell whether $* 5 B-d$ would be independent or dependent.
} 
(41) From tetrameter to hexameter

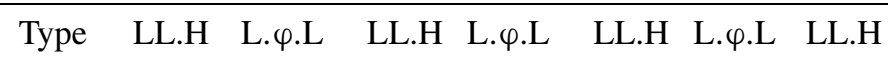

\begin{tabular}{|c|c|c|c|c|c|c|c|}
\hline $\mathrm{E}$ & $2 \mathrm{~A}-\mathrm{a}$ & L.H .L LL.H & L.H .L LL.H & & & & independent \\
\hline $\mathrm{E}$ & $3 \mathrm{~A}-\mathrm{c}$ & L.H .L LL.H & L.H .L LL.H & L. $\varnothing . \varnothing$ & & & independent \\
\hline $\mathrm{E}$ & $3 \mathrm{~A}-\mathrm{b}$ & L.H .L LL.H & L.H .L LL.H & L.H.Ø & & & independent \\
\hline $\mathrm{E}$ & $* 3 \mathrm{~A}-\mathrm{a}$ & L.H .L LL.H & L.H .L LL.H & L.H.L & & & endlapse \\
\hline $\mathrm{E}$ & $4 \mathrm{~A}-\mathrm{c}$ & L.H .L LL.H & L.H .L LL.H & L.H.L & LØ.Ø & & ident \\
\hline E & $4 \mathrm{~A}-\mathrm{b}$ & L.H .L LL.H & L.H .L LL.H & L.H.L & LL. $\varnothing$ & & dent \\
\hline $\mathrm{O}$ & 7FC-ii $\varnothing \varnothing . H$ & L.LL.L LL.H & L.LL.L LL.H & L.H.L & LL. $\varnothing$ & & independent \\
\hline $\mathrm{E}$ & $* 4 \mathrm{~A}-\mathrm{a}$ & L.H .L LL.H & L.H .L LL.H & L.H.L & LL.H & 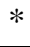 & gap \\
\hline
\end{tabular}

Note that 7FC-ii, the longest meter in the system, is crucially fitted to six verse feet. This meter is sorted as an $\mathrm{O}$ meter by Dell and Elmedlaoui, since the first foot is a catalectic version of LL.H. The corresponding, acatalectic meter of six verse feet (*4A-a) is missing. It is clearly on the margin of the system, lengthwise, but we have no particular reason to assume that it should be excluded in principle, as several of the extant meters end in LL.H.

The last two illustrations of transitions show rightward shifting tetrameter and dimeter. We start from the missing lapsing tetrameter, *6A-a, and list the crucially fitted tetrameters.

(42) Shifting tetrameter

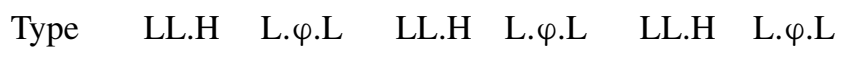

\begin{tabular}{|c|c|c|c|c|c|c|c|c|}
\hline $\mathrm{O}$ & $* 6 \mathrm{~A}-\mathrm{a}$ & $L L . H$ & $L . H . L$ & LL.H & $L . H \quad . L$ & & & * endlapse \\
\hline $\mathrm{O}$ & 6FB-i & $\varnothing \mathrm{L} . \mathrm{H}$ & L.LL.L & LL.H & L.H .L & LØ.Ø & & independent \\
\hline $\mathrm{O}$ & 6FC-i & $\varnothing \mathrm{L} . \mathrm{H}$ & L.LL.L & LL.H & L.LL.L & LØ.Ø & & independent \\
\hline $\mathrm{O}$ & 6FA-iii & Ø.Н & L.H .L & LL.H & L.H .L & LØ.Ø & & independent \\
\hline $\mathrm{O}$ & 6FB-iii & ØØ.Н & L.LL.L & LL.H & L.H .L & LØ.Ø & & independent \\
\hline $\mathrm{O}$ & 6FC-iii & ØØ.Н & L.LL.L & LL.H & L.LL.L & LØ.Ø & & independent \\
\hline $\mathrm{O}$ & $6 \mathrm{FC}-\mathrm{ii}$ & ØØ.Н & L.LL.L & LL.H & L.LL.L & LL. $\varnothing$ & & independent \\
\hline E & $2 \mathrm{~A}-\mathrm{a}$ & & L.H .L & LL.H & L.H .L & LL.H & & independent \\
\hline E & $2 \mathrm{~B}-\mathrm{a}$ & & L.H .L & LL.H & L.LL.L & LL.H & & independent \\
\hline E & $2 \mathrm{FA}-\mathrm{i}$ & & Ø.H .L & LL.H & L.H .L & LL.H & L.Ø.Ø & independent \\
\hline E & 2FB-ii & & Ø.Ø .L & LL.H & L.LL.L & LL.H & L.H.Ø & independent \\
\hline $\mathrm{E}$ & 2FA-ii & & Ø.Ø .L & LL.H & L.H .L & LL.H & L.H.Ø & independent \\
\hline
\end{tabular}

As can be seen, the $\mathrm{O}$ type meters shift seamlessly into $\mathrm{E}$ type meters, which indicates that the division into $\mathrm{O}$ and $\mathrm{E}$ type meters is not basic, but just a descriptive labelling. Note that it is only if we recognize the fitted count that the seamless transition is revealed. The shifting dimeters are given in (43). 
Shifting dimeter

\begin{tabular}{|c|c|c|c|c|c|c|c|}
\hline & Type & LL.H & L. $\varphi . L$ & LL.H & L. $\varphi . \mathrm{L}$ & & \\
\hline $\mathrm{O}$ & $* 11 \mathrm{~A}-\mathrm{a}$ & $L L . H$ & L.H .L & & & * & endlapse \\
\hline $\mathrm{O}$ & 11FB-i & $\varnothing \mathrm{L} . \mathrm{H}$ & L.LL.L & Lø.Ø & & & dependent \\
\hline $\mathrm{O}$ & 11FA-ii & $\varnothing \varnothing . H$ & L.H .L & LL. $\varnothing$ & & & dependent \\
\hline $\mathrm{O}$ & 11FB-ii & $\varnothing \varnothing . Н$ & L.LL.L & LL. $\varnothing$ & & & dependent \\
\hline $\mathrm{E}$ & $9 A-a$ & & L.H .L & LL.H & & & dependent \\
\hline $\mathrm{E}$ & 9FA-i & & Ø.H.L & LL.H & L. $\varnothing . \varnothing$ & & dependent \\
\hline $\mathrm{E}$ & 9FB-ii & & $\varnothing . \varnothing . \mathrm{L}$ & LL.H & L.LL.O & & dependent \\
\hline $\mathrm{O}$ & $* 11 \mathrm{~B}-\mathrm{a}$ & & & LL.H & L.LL.L & * & endlapse \\
\hline
\end{tabular}

We only find this shifting pattern with tetrameters and dimeters, testifying to the relative unmarkedness of these lengths (cf. Sect. 6.2 below).

Looking over the whole set of meters, we have found that they circumscribe a coherent region of the available metrical space. The meters all have immediate, minimally different, neighbours. The gaps that we have found inside the system are primarily due to the operation of NOLAPSE in final anaclastic verse feet. An example is repeated in (44).

Systematic gap

\begin{tabular}{|c|c|c|c|c|c|c|}
\hline & Type & L. $\varphi . \mathrm{L}$ & LL.H & L. $\varphi . \mathrm{L}$ & & \\
\hline$E$ & $1 \mathrm{~A}-\mathrm{b}$ & L.H.L & LL.H & L.H. $\varnothing$ & & inde \\
\hline $\mathrm{E}$ & $* 1 \mathrm{~A}-\mathrm{a}$ & L.H.L & LL.H & L.H.L & $*$ & endlapse \\
\hline $\mathrm{E}$ & $10 \mathrm{~A}-\mathrm{d}$ & Ø.H.L & LL.H & L.H.L & & dependent \\
\hline
\end{tabular}

Beside the systematic gaps there is only one accidental gap, inside the system (i.e. with neighbours on either side), that is unaccounted for, namely $* 5 B-d$, repeated below with its immediate neighbours.

Accidental gap

\begin{tabular}{|c|c|c|c|c|c|c|}
\hline & Type & LL.H & L. $\varphi . \mathrm{L}$ & LL.H & & \\
\hline $\mathrm{O}$ & $5 B-a$ & LL.H & L.LL.L & LL.H & & independent \\
\hline $\mathrm{O}$ & $* 5 B-d$ & $\varnothing L . H$ & L.LL.L & $L L . H$ & $*$ & gap \\
\hline $\mathrm{O}$ & 12A-x & ØØ.Н & L.H .L & LL.H & & dependent \\
\hline
\end{tabular}

We would not be surprised if this meter were found under further data collection. We have noted some meters as missing on the margin. There is of course an endless number of possible meters if one lets the meter system grow in line length, though this might interfere with musical constraints. We cannot, at this point, evaluate the status of potential meters on the margin in any meaningful way.

The variation between L.H.L and L.LL.L has proven to be somewhat unpredictable. It is not the case that for every meter that contains a L.LL.L in some position, there will be a corresponding meter with L.H.L in the same position. For instance, in cluster 6 there were more meters with L.LL.L in the second verse foot, than me- 
ters with L.H.L in that position. But only L.H.L can begin an independent meter. We maintain, with Dell and Elmedlaoui, that L.H.L is more basic, and that the variations between L.H.L and L.LL.L within clusters are due to other constraints. It might be the case that variants partially block each other, by being so similar. Deeper study of these matters, including properties of the text and the music might shed more light on the issue.

\subsection{Preference for binarity}

Looking over the 56 meters in the corpus, we find that there are biases regarding length. The preferred length is four verse feet for independent meters and two verse feet for dependent meters. Hence, relative binarity is favoured at the line level and probably one level down, if dependent meters are taken to be smaller units than full lines. For tetrameter, the bias for binarity shows already in the structural count, but it is much more pronounced in the fitted count. For the dependent meters, it is only the fitted count that reveals the preference for dimeter. The figures for the two manners of counting are given in (46) with the fitted counts centred.

Structural and fitted counts of meters

\begin{tabular}{|c|c|c|c|c|}
\cline { 2 - 5 } \multicolumn{1}{c|}{} & \multicolumn{2}{c|}{ independent meters } & \multicolumn{2}{c|}{ dependent meters } \\
\hline $\begin{array}{c}\text { number of verse } \\
\text { feet per meter }\end{array}$ & $\begin{array}{c}\text { structural } \\
\text { count }\end{array}$ & $\begin{array}{c}\text { fitted } \\
\text { count }\end{array}$ & $\begin{array}{c}\text { fitted } \\
\text { count }\end{array}$ & $\begin{array}{c}\text { structural } \\
\text { count }\end{array}$ \\
\hline one & - & - & 1 & 1 \\
\hline two & - & - & $\mathbf{1 7}$ & 9 \\
\hline three & 3 & 3 & 3 & 11 \\
\hline four & 18 & $\mathbf{2 7}$ & - & - \\
\hline five & 11 & 2 & - & - \\
\hline six & 2 & 3 & - & - \\
\hline seven & 1 & - & - & - \\
\hline total & 35 & 35 & 21 & 21 \\
\hline
\end{tabular}

Under each count, the meters containing four verse feet constitute the largest group. In the fitted count the number of independent meters containing four verse feet increases radically (from 18 to 27 ), while meters containing five verse feet in the structural count reduce from 11 to 2 . The single independent meter of 7 verse feet in the structural count comes out as one of 6 verse feet in the fitted count. These figures point at the relevance of the fitted count, which strengthens the generalization that unmarked tetrameter is preferred. In the dependent, generally shorter meters, the fitted count centres on 2 verse feet. As shown above in (38) and (39), the independent and dependent meters are not categorically different, and so they should be treated in a way that heeds the continuum.

Binarity is also the rule at the level of the verse foot as well as the metrical position. Verse feet inside meters are invariably four moras. The only situation in which binarity is broken is at the end of lines, where catalexis may apply. The metrical system is hence binary to a very high degree. In many other systems, some binarity is regularly broken inside meters in order to create or abide by some other regularity. For instance, in the iambic trimeter of Ancient Greek meter, every metron contains 
a stable light syllable in the third position (e.g. HH.LHH, HLL.LLH, Golston and Riad 2000). Also, the metra in all meters of Classical Arabic always contain exactly one light syllable (Golston and Riad 2016), and several of these meters also have regularly missing positions in every other metron. We also find lines that depart from binary lengths in both Greek and Arabic, e.g. trimeter. These properties all entail breaches of binarity at various levels, as part of the specification of individual meters.

The Tashlhiyt Berber system therefore appears binary to a higher degree than many other systems, and this unmarked property emerges in the metrical system, much as unmarked properties tend to emerge in prosodic morphology. This fact carries implications that we turn to next.

\subsection{Emergent line length}

The Tashlhiyt meters are not basically specified as trimeter, tetrameter, pentameter, etc. Rather it would seem that line length is emergent. Two of the observations above lead to this conclusion. First, there is a preference for binary lengths in the meters, cf. (46), a fact that in itself testifies to the emergence of the unmarked rather than to a principled specification of line length. The fact that the lower units (metrical position, verse foot) are binary, too, provides additional support. Second, there are no obvious cut-off points between meters regarding overall length, cf. (38)-(41). When we looked at the transitions between the individual meters we found that they were all stepwise. The straight meters form a large coherent family, where the variation in length is modulated in minimal steps, one syllable or metrical position at a time. If line length were regulated at the level of the abstract metrical schema, then we should have been able to observe families based on length in terms of verse feet. As the case is, attempts at breaking them up into basic dimeters, trimeters, tetrameters, etc. would be artificial. Each individual meter that departs from tetrameter and dimeter must still be specified for length and we will propose a way to do that in Sect. 7.3.

Emergent line length has implications for the theory of metrics. If line length is not fixed in a principled manner in the abstract metrical schema for the straight meters (as under the analysis given here), then counting cannot be basic in Tashlhiyt Berber meter. This would go counter to the claim in Fabb and Halle (2008) for meter in general, where the line as such and its measure are the defining characteristics of verse. Even if this is atypical, it makes Tashlhiyt a challenge to theories of meter that claim that counting and/or line length is basic. Tashlhiyt meter hence also goes against the spirit of one of the so-called structure parameters (NUMBER OF FEET) of Hanson and Kiparsky (1996), and pretty much all traditional work on metrics. At the very least, it means that for verse systems that have an abstract general schema from which individual meters are drawn, one aspect of the abstractness could be the underspecification of line length, along with underspecification of certain metrical positions (e.g. anceps ' $\sigma$ ' as L or H, or prosodic feet ' $\varphi$ ' as H or LL, in Greek, Latin and Arabic), and ordering variation (as ' $E$ ' or ' $O$ ' in the Tashlhiyt system). The Sanskrit meters analyzed in Deo (2007) all have prespecified line length at the abstract level of the metrical schema. Tashlhiyt appears to show that this is a variable that is subject to underspecification at the abstract level. Instantiations of the metrical schema will manifest a length, however, and this length has to be the same throughout a poem due 
to the generalized responsion. The question then is how the preferred lengths that we find are motivated.

If line length does not follow from a stipulation, a parameter setting, or such, then two sources for emergent binary line length seem close at hand. The first one would be music, and the second language. ${ }^{27}$ We will call these the musical hypothesis and the linguistic hypothesis. Of these, we are unable to evaluate the musical hypothesis without further study, but will repeat some of the observations made in Dell and Elmedlaoui (2008). They note that there is a relationship between line length and musical length. Stanzas and half-stanzas in Tashlhiyt songs must minimally fill out four tactus beats (Dell and Elmedlaoui (2008: 85) ${ }^{28}$ A verse foot can accommodate no more than one beat, which occurs on the second mora (Dell and Elmedlaoui's BEAT, 2008: 40ff.). Dell and Elmedlaoui take the requirement of four tactus beats as their criterion for separating independent and dependent meters. Thus, two dependent meters may combine to make a sufficiently long string to fit into a four beat stanza. Complementarity need not be symmetrical, which means that it is fine to combine units of different lengths into stanzas. Often, however, complementarity is symmetrical. Thus, there could be some connection between music and meter which might bias things towards binarity, even though independent meters may contain as few as three verse feet (cf. 1Ab and 1Ac in (38)). Another fact that would likely have a connection with the alignment of meter to music is the (crucially) fitted count. Where regular catalexis suppresses syllables (analyzed as distinctive binarity violations in Sect. 7.3), a crucially fitted count doesn't (necessarily) suppress anything, it only shifts the starting point. This fact would appear to be extralinguistic. However, while the alignment of verse feet with the musical beat is regular, the exact line length is not obviously predictable from the music as such. At any rate, it is fair to say that the preference for tetrameter could be due, ultimately, to the alignment of meter with music.

The second hypothesis would be that binary lengths emerge from linguistic grammar. The linguistic hypothesis proposes that it is the binarity of prosodic structure that is expressed in meter. Binarity as such could well stem from universal grammar (like any constraint in an Optimality Theory grammar, Prince and Smolensky 1993), but the way it plays out in a given language will be subject to the language-specific grammar, in this case that of Tashlhiyt Berber. The important thing for our purposes is that these constraints are present in the linguistic grammar, and that they could thereby be employed, potentially, in a model of the meter/language relationship, without making a detour (as it were) to a meter-making component (Hanson and Kiparsky 1996; Blumenfeld 2015). This should give us a model with less duplication of information, e.g. in the binarity area, and one which makes predictions regarding the languagespecific influence on meter. If meters are made in a universal meter-making component, the language-specific influence can hardly be predicted without stipulation at some stage in the analysis. If we can go directly from the ordinary, linguistic grammar

${ }^{27}$ The third possibility is specification within a universal metrics module as generally assumed in Hanson and Kiparsky (1996) and Blumenfeld (2015). In view of the fact that we are looking at realizations of a meter, rather than the basic meter, this option seems less attractive and will not be pursued here.

${ }^{28}$ There is some room for pauses, so this requirement does not mean that there must be strictly four verse feet. 
to the meter, that would be a stronger model. This is the more conservative scenario that we pursue here. ${ }^{29}$

\section{The homology of prosody and meter}

The hypothesis of the homology of the categories of prosody and meter that we presented in Sect. 2 entails that the notions of 'metrical position', 'verse foot', 'dipody', 'halfline', and 'line' are all just parallel terms for the categories of the prosodic hierarchy: 'syllable', 'prosodic foot', 'prosodic word', 'prosodic phrase', and 'intonation phrase'. Exactly how the parallel plays out in an individual language will depend on the grammar of that language. In Tashlhiyt, the metrical position is a prosodic foot, rather than a syllable, whereas in English, the metrical position will typically be a syllable. These are facts about the prosody of these languages, not about metrics specifically.

By the same token, the hypothesis avoids duplication and provides some predictions. Duplication is avoided if our base line is that the categories of meter and those of the prosodic hierarchy are not distinct. The general prediction is that the categories of meter should be recognizable from the language. In practise this will typically concern the lower levels of the prosodic hierarchy where basic categories of prosody are more easily recognized (mora, syllable, foot, prosodic word) and where many languages exhibit so-called prosodic morphology (reduplication, truncation, nickname formation, root-and-pattern templates), which often exposes the canonical size of prosodic categories. For Germanic languages like German and Swedish, the similarity regarding prosodic words is found in the shape of nicknames (German Hansi, Biggi for Hans, Brigitte; Swedish Kattis, Svempa for Katarina, Sven), on the one hand, and the shape of verse feet of the most unmarked kind, trochees based on syllables, on the other. Thus, the homology of the trochee in verse and the trochee in nicknames in German and Swedish amounts to an argument for the homology of the prosodic word and the verse foot. In Arabic, one finds a preference for an iambic shape in verse feet (Fleisch 1956/1968; Golston and Riad 1997), as well as in some of the prosodic morphology of the language, namely broken plurals and diminutives (McCarthy and Prince 1990). In Tashlhiyt, too, there are indications of a correspondence between meter and prosodic morphology. Thus, we find the prosodic shapes LL (a moraic trochee) and L.H.L (a prosodic word) not only in the straight meters, but also in several prosodic morphemes: Imperfective, Tifrdi, Tirrugza, Abnakliy, Azddayru (Dell and Elmedlaoui 1992/2001, 2002, 2013; Jebbour 1999; Bensoukas 2001). Scholars are, however, not fully agreed on how the analyses of the prosodic morphemes should be carried out, and a fuller analysis of the parallels with meter will have to wait. The similarity of verse feet and prosodic morphemes might be coincidental, but the more attractive hypothesis is that they are exponents of the same facts about preferred prosody. Meter as well as prosodic morphology tend to exhibit unmarked structure (e.g. McCarthy and Prince 1986;

\footnotetext{
${ }^{29}$ We might add that the linguistic hypothesis is not in conflict with the musical hypothesis. The two structures are separate, and the relevance of musical structure could be diachronic, while a linguistic analysis is warranted for the structural synchronic analysis.
} 
Downing 2006: 5ff.; Blumenfeld 2015). This shows up in properties like binarity, eurhythm and (in prosodic morphology) the avoidance of syllabic complexity. The source for these unmarked properties is usually taken to be the grammar itself. Marked properties are lexically specified, as usual. All that we have assumed here is that this line of reasoning should be extended to the higher categories of the prosodic hierarchy, where it yields meter.

Prosodic categories higher up are larger than typical morphemes and so they will not be found in prosodic morphology as such. However, there are other indications that make the connection between the prosodic hierarchy and meter natural at the higher levels. Prosodic binarity at the level of the prosodic phrase and the intonation phrase appears in several languages, in the face of syntactic alignment (Itô and Mester 2006 for Japanese; Prieto 2006 for Spanish; Selkirk and Elordieta 2010 for Basque; Bennett et al. 2016 for Irish; Frota and Vigário 2007 for Portuguese). Also, there are phenomena that indicate that reduplication of large units is possible in principle (Ghomeshi et al. 2004). While meter is not a type of reduplication, the latter argument tells us that size is not in itself an obstacle to prosodic copying.

The general proposal is that meter is derived in the same way as prosodic morphemes, where the similarity is the greatest with root-and-pattern templates. For prosodic morphemes the general reason for the emergence of unmarkedness is the fact that the prosodic structure is unfettered by faithfulness and (some) alignment. The segmental content comes from elsewhere (by copying, epenthesis or by simultaneous representation, see Sect. 7.1) so there is no segmental structure to be faithful to. ${ }^{30}$ The morpheme is just prosodically specified, either directly as a prosodic category (McCarthy and Prince 1986), or as a morphological category which receives a prosodic form via the grammar (McCarthy and Prince 1994). Segmental material will either be copied into it or supplied by the grammar (epenthesis), or the prosodic morpheme will get to regiment all or part of the segmental material in parallel with the regular prosody. Like faithfulness, alignment of prosody to morpho-syntax is usually rather high-ranking in grammars, and therefore the binarity of categories like the prosodic phrase and the intonation phrase is often not very clearly seen in regular grammar (but see e.g. Bennett et al. 2016). The emergent binarity seen in prosodic morphemes and, we claim, in meter, stems from the linguistic constraints that mandate binarity, not from some external source. These wellformedness constraints dominate the constraints that require the prosodic morpheme to be (segmentally) identical to the base, and the constraints that align prosodic categories with morpho-syntactic ones. This is illustrated in (47).

$$
\begin{array}{ccc}
\text { Emergence of binarity in prosodic morphemes and meter } \\
\text { Input } \leftrightarrow \text { Output } & \text { Base } \leftrightarrow \text { Prosodic morpheme } \\
\text { faithfulness } & \gg \text { Wellformedness } \gg & \text { identity } \\
\begin{array}{c}
\text { Syntax } \leftrightarrow \text { Prosody } \\
\text { alignment }
\end{array} & \text { (binarity, eurhythm) } & \text { Base } \leftrightarrow \text { Meter } \\
\text { alignment }
\end{array}
$$

In the regular case, prosodic structure aligns with morpho-syntactic structure, with morpho-syntax calling the shots. Binarity shines through only under favourable cir-

\footnotetext{
${ }^{30}$ Provision has to be made here for root-and-pattern morphology where the prosodic morpheme may come with a particular vocalism (e.g. the Arabic diminutives in (50)).
} 
cumstances. The relationship between meter and morpho-syntax is much more variable, and often irrelevant. I have referred to it as Base $\leftrightarrow$ Meter alignment in (47), where the base is the text. ${ }^{31}$ The Base $\leftrightarrow$ Meter alignment is dominated by wellformedness constraints on binarity and eurhythm, which give the meter its general form, just like in other prosodic morphemes. If we take the generalized template approach of McCarthy and Prince (1994), prosodic morphemes are in fact morphological categories (stem, prefix) which get their unmarked prosodic shape from the grammar. Our view of meter as an extension of prosodic morphology is illustrated in (48) and (49), where the division into (a) and (b) is just descriptive of prosodic morphology and meter.

Morphosyntactic categories

a. ReduPliCANT $=$ Stem/Prefix

NICKNAME $=$ Stem/Prefix

b. $\mathrm{METER}=\mathrm{Clause} /$ Sentence

DIPODY/COLON/METRON $=$ XP

VERSEFOOT $=$ Stem

(49) Morphosyntactic alignments

a. $\quad$ Stem $\leftrightarrow$ Prosodic Word

Prefix $\leftrightarrow$ Foot

Prefix $\leftrightarrow$ Syllable

b. Clause/Sentence $\leftrightarrow$ Intonation Phrase

$\mathrm{XP} \leftrightarrow$ Prosodic Phrase

Stem $\leftrightarrow$ Prosodic Word

We will not review the analytical possibilities for prosodic morphology in detail here (see e.g. Downing 2006; Flack 2007; Downing and Mous 2011), but be content to state that whatever analysis one chooses for prosodic morphemes should also be used for meter, the important fact being that they come from the same linguistic source, namely the grammar in combination with any marked lexical information.

The particular properties of meter that set it apart from prosodic morphology are, first, that meter is made from the higher categories of the prosodic hierarchy (typically the intonation phrase or the prosodic phrase). The higher categories of the prosodic tree naturally contain the lower categories such as prosodic feet and prosodic words, for which the equivalence with metrical positions and verse feet is easier to detect. This makes meter a type of prosodic morpheme with greater complexity than the more familiar prosodic morphemes. This richness of structure also allows for (marked) modulation of categories at different levels, which is how more complex meters can be created (cf. for example the use of catalexis at different levels in the Classical Arabic meters tawīl, basīt, and madīd, where every other metron is catalec-

${ }^{31}$ The nature of this alignment is typically rather rudimentary in stress languages, with lines often beginning and ending where there are major syntactic boundaries, but without heeding smaller ones. 
tic, beside optional catalexis at the halfline and line levels, Golston and Riad 1997, 2016).

Another property of meter which sets it apart also from at least nicknames and reduplication is the fact that the prosodic template is simultaneous with the regular prosodic structure. In this particular respect, meter is the most similar to root-andpattern morphology. We turn to this in the next section.

\subsection{Simultaneous prosodic structures}

While a nickname morpheme may supplant the regular morpheme and its prosody, and the process of reduplication adds a morpheme next to the regular base morpheme and its prosody, root-and-pattern morphemes are realized simultaneously with the regular word and its prosody. This is also the case with meter and the regular prosody, cf. (1) and (5). Let us now look at the simultaneous prosodic structures that are found in prosodic morphology of the root-and-pattern type. Arabic broken plurals and diminutives, and Tashlhiyt so-called Tifrdi and Tirrugza nouns are relevant examples, where the template and the full word may have different sizes. The Arabic cases are illustrated in (50), cf. McCarthy and Prince (1990).

Arabic broken plural and diminutive formation

\begin{tabular}{|c|c|c|c|c|c|c|}
\hline Root & Singular & Gloss & $\begin{array}{l}\text { Target } \\
\text { shape }\end{array}$ & $\begin{array}{l}\text { Plural } \\
\text { word }\end{array}$ & $\begin{array}{l}\text { Diminutive } \\
\text { word }\end{array}$ & $\begin{array}{l}\text { Word } \\
\text { shape }\end{array}$ \\
\hline nfs/ & nafs & 'soul' & LH & {$[\mathrm{nu}$} & & LH \\
\hline /rjl/ & rajul & 'man' & LH & [rijaal] & [rujayl] & LH \\
\hline xtm/ & xaatam & 'signet ring' & LH & [xawaa]tim & [xuway]tim & LHH \\
\hline /jndb/ & jundub & 'locust' & LH & [janaa]dib & [junay]dib & LHH \\
\hline
\end{tabular}

We leave vocalism to the side and look only at the prosodic shapes. The prosodic shape of the target for both of these formations is LH, while the actual output word may be the same size or longer. This in turn indicates that there are two prosodic structures involved, one for the broken plural and one for the word in its entirety. The Tashlhiyt cases are illustrated in (51) and (52). Dell and Elmedlaoui (1992/2001: 563ff., 543) and Jebbour (1999: 111) provide a long list of forms for this and several other templatic derivational processes. ${ }^{32}$ Moraic segments are underlined.

(51) Tifrdi deverbal noun formation

\begin{tabular}{|c|c|c|c|c|c|c|}
\hline Base & Gloss & $\begin{array}{l}\text { Tifrdi } \\
\text { stem }\end{array}$ & $\begin{array}{l}\text { Target } \\
\text { shape }\end{array}$ & $\begin{array}{l}\text { Tifrdi } \\
\text { word }\end{array}$ & $\begin{array}{l}\text { Word } \\
\text { shape }\end{array}$ & gloss \\
\hline frd & 'graze' & fr.di & LL & $\mathrm{t}-\underline{\mathrm{i}}$-frddi & LLL & 'grazing' \\
\hline $\mathrm{nm}$ & 'be straight' & n.mi & LL & $\mathrm{t}-\underline{\mathrm{i}-\mathrm{nm}} \underline{\mathrm{i}}$ & $\mathrm{HL}$ & 'straightness' \\
\hline
\end{tabular}

${ }^{32}$ Unlike the case in Arabic, radical sequences may contain vocalic elements (Dell and Elmedlaoui 1992/2001: 90). 
Tirrugza nouns

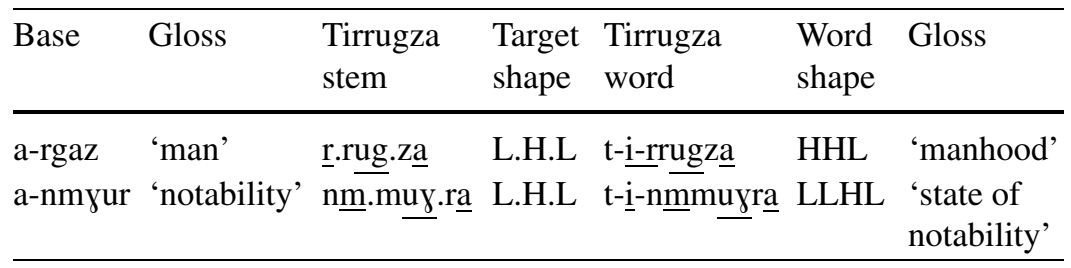

The templatic part of the word formation processes in Tashlhiyt concerns the stem, which is here [LL] for Tifrdi (a prosodic foot), and [LHL] for Tirrugza (a prosodic word, of a shape that we recognize from meter). In addition to the stem there is a prefixed syllable $t-i$ - which parses as either a light syllable or as a heavy syllable together with the first radical consonant, depending on sonority and syllabification (Dell and Elmedlaoui 2002). The simultaneity of the two structures is illustrated in (53) and (54), where regular prosody is on top, and the templatic requirement below.

Tifrdi deverbal noun formation
( $\left.\begin{array}{lll}\mathrm{L} & \mathrm{L} & \mathrm{L}\end{array}\right)$
$\left(\begin{array}{ll}\mathrm{H} & \mathrm{L}\end{array}\right)$
Prosodic structure
$\mathrm{t}$ i f r d i
t i n $\mathrm{m} \mathrm{i}$
[L L]
$\left[\begin{array}{ll}\mathrm{L} & \mathrm{L}\end{array}\right]$
Prosodic morphology

(54) Tirrugza nouns
$\left(\begin{array}{lll}\mathrm{H} & \mathrm{H} & \mathrm{L}\end{array}\right)$
( $\mathrm{L} \mathrm{L}$
H L) Prosodic structure
t irrugza
t i n m m u r a

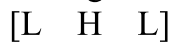
$\left[\begin{array}{lll}\mathrm{L} & \mathrm{H} & \mathrm{L}\end{array}\right]$
Prosodic morphology

Root-and-pattern-like formations represent the situation where the realization of the prosodic template is simultaneous (but not coextensive) with a regular prosodic structure. The point of this in the present context is that it shows that nothing prevents two prosodic structures from being co-present in a single form, in prosodic morphology. This is crucial to our analysis of meter as grammatically controlled. We have seen the situation where the two prosodic structures are not entirely coextensive also in meter, where in (1) there was an extrametrical syllable at the end of the line and in (5) where all of four syllables were extrametrical, two in each halfline. In these cases, the regular prosody prosodifies all text, whereas the meter is regulated in length such that a difference occurs which could be described as extrametricality (from the perspective of the meter).

The simultaneity of the prosodic template with the regular prosody is tacitly or explicitly assumed in most analyses of meter. The usual assumption is that the metrical template is external, conceived of as an artefact (traditional metrics) or a structure generated from a universal meter module with some kind of principled relation to language (generative metrics). The template is then matched to prosodic properties of the text (Hanson and Kiparsky 1996; Blumenfeld 2015). The matching of the template and the prosody is illustrated below with an example of iambic pentameter, very slightly adapted from Blumenfeld (2015: 93). The prosodic structure is on top and the metrical template below the text (Robert Frost, A boy's will, Waiting, line 21). 
Iambic pentameter, Blumenfeld (2015)

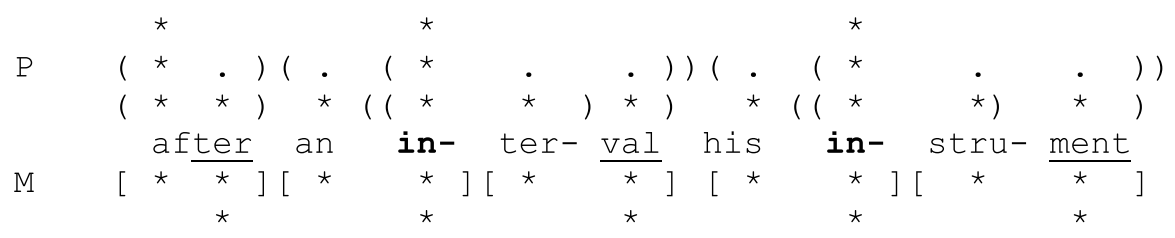

In much earlier work the template is given as a tree structure with nodes marked $\mathrm{S}$ or W, for 'strong' and 'weak', respectively. Here, the template has the shape of a grid. The overall correspondence between meter and prosody is a matter of degree, and allowance has to be made for what is acceptable as a line of iambic pentameter. ${ }^{33}$ Some stressed syllables coincide with strong positions in the template (bolded), but also some stressless syllables are matched to strong positions in the template (underlined). Other lines may have complete agreement between stressed syllables and $\mathrm{S}$ positions, and a very few lines will have no stressed syllable in strong positions. In Blumenfeld's model these things are evaluated in terms of faithfulness (i.e. similarity) between the two prosodic structures that are matched: "Lines are metrical to the extent that their prosodic structure reproduces the template. A line which deviates from the prosody of the template too far can be deemed unmetrical." (Blumenfeld 2015: 85). This procedure may be required to capture certain kinds of meters, in particular those where gradient wellformedness is in evidence, such as English iambic pentameter. However, it is an inevitable consequence that the relationship between meter and language in such an analysis is less direct, and less linguistic, than in the model proposed here for Tashlhiyt meter where the meter is constituted by constant properties.

In the model advocated here, the metrical template is generated by the grammar itself rather than by a universal meter module. A few things follow from this assumption. For one thing, it means that the unmarked properties of meter (such as binarity) will manifest themselves in the same way as they do for prosodic morphemes, see (47). Also, the content of metrical positions will be the same prosodic categories that occur in the language. In our model this follows from the homology of meter and prosody. The fact that the grammar is the source for the metrical tree also predicts that there will be no prespecified rhythmic content in terms of S/W structure in meter, since that type of information is not linguistic. Prominence at some edge will be a question of alignment, as in ordinary language. ${ }^{34}$ The absence of $\mathrm{S}$ and $\mathrm{W}$ thus does not deny headedness in prosodic categories, only that categories of meter should come with predefined prominence.

\footnotetext{
${ }^{33}$ In the standard analysis of the iambic pentameter of Shakespeare (sonnets), the requirement is for $\mathrm{W}$ positions not to contain stressed syllables of polysyllabic words (except in phrase-initial positions, where inversion is permitted).

${ }^{34} \mathrm{The} \mathrm{S} / \mathrm{W}$ structure is perhaps the most distinctive feature of the generative approach to meter, with a template originating outside linguistic grammar. Many other things, like the acknowledgement of the linguistic control over what goes in metrical positions, are only technically distinct from the approach here.
} 


\subsection{Improvement in Tashlhiyt Berber metrics}

We claimed in Sect. 2.1 that NoCLASH is the privileged constraint that defines the straight meter family in Tashlhiyt. Given the preceding discussion, we can now put this proposal into an analytical context. NoCLASH expresses a linguistic desideratum which is obeyed in straight meters, but which is often violated in the ordinary language. By making this constraint privileged, we get a meter that contains no clashes, entailing overall improvement in that particular respect. There are consequences in terms of anaclasis (LLH realized as LHL), and perhaps even increased violation of NOLAPSE in meter compared with prose. But these things are unimportant, and not evaluated under the hypothesis of privileged constraints. The regular prosodic tree sees to it that whatever text is produced gets a prosodic structure that functions for all linguistic purposes. The meter only obeys the privileged constraint, instantiating an improvement in the particular respect that this constraint demands. ${ }^{35}$ There could be different ways to comply with the requirement of the privileged constraint. Thus, NoCLASH is equally met whether some verse feet are forced to be anaclastic (LHL) or some metrical positions are unary, as in the case of trochees (HL.HL). Under the hypothesis of privileged constraints, alternatives of compliance are not evaluated in meter. ${ }^{36}$ However, grammar will likely have a say here, by (in this case) not upsetting the unmarkedness regarding binarity that emerges in the prosodic morpheme. With other privileged constraints the outcome could be different.

Binarity comes about by the grammatical emergence of the unmarked in prosodic morphemes (cf. (47)). Inside Tashlhiyt meters, binarity is always realized at the levels of the prosodic foot (LL, H) and prosodic word (LLH, LHL). At the levels of the prosodic phrase and the intonation phrase, binarity is preferred as seen in the overview in (46), where independent and dependent meters tend to contain four and two verse feet, respectively. We take this to mean that the basic meter does not have a specified line length, but that its length emerges. However, line length does vary in the individual instantiations of the basic meter and the mechanism that instantiates this variation is catalexis. Catalexis imposes markedness and is clearly part of the lexical specification of a meter. In the next section we turn to the formal status of catalexis.

\subsection{Distinctive violation in Tashlhiyt meter}

The basic underlying form for the straight meter is METER=Clause, as given in (48). ${ }^{37}$ The grammar associates the clause with an intonation phrase, cf. (49). The binarity constraints yield an unmarked line of four verse feet (=prosodic words), each containing a two metrical positions (=prosodic feet, ' $\varphi$ '). Verse feet are organized into two dipodies/halflines (=prosodic phrases) which in turn are organized into a line (=intonation phrase). (56) illustrates the most unmarked of the straight

\footnotetext{
${ }^{35}$ There is no reason of principle why not more than one constraint could be privileged for a given meter.

${ }^{36}$ This amounts to saying that there is no separate grammar with its own internal constraint ranking in meter, and hence no separate evaluation of constraint violation (in such a grammar).

${ }^{37}$ Dependent meters could be considered prosodic phrases (rather than intonation phrases), where the emergent length is two verse feet.
} 
meters. ${ }^{38}$ The effect of the privileged constraint NOCLASH is registered below the tree.

(56) Binarity of Tashlhiyt Berber meter: 2A-a

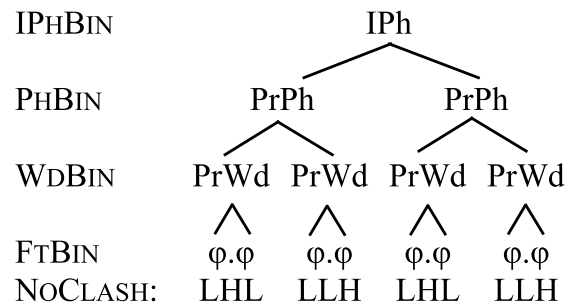

Catalexis involves the suppression of syllables at edges of lines. This means that peripheral verse feet will be lacking one or two syllables, and we will analyze this as a required violation of BINARITY. To get the meter called 2A-b, the last $\mathrm{H}$ syllable should be suppressed. This entails a violation of WORDBINARITY, as illustrated in (57) where the upper part of the tree (identical to (56)) has been removed.

Binarity and catalexis: $2 \mathrm{~A}-\mathrm{b}$
WDBIN

Catalexis is what makes 2A-b distinct from 2A-a and so it must be lexically specified. We can formally express catalexis as a distinctive (i.e. required) violation of the constraint WDBIN (for 2A-b). In this, we follow Golston (1996) who proposes that all underlying information is expressed in terms of distinctive violations of constraints. For this instantiation of a straight meter, then, the specification would look something like (58).

Straight meter $2 \mathrm{~A}-\mathrm{b}$

METER $=$ Clause

Straight meter: NoCLASH is privileged

2A-b: WDBIN is distinctively violated once

The class of straight meters is defined by the privileged status afforded to NoCLASH. Given that meter is a clause combined with the workings of unmarkedness, an allbinary meter of four verse feet is provided by grammar, i.e. 2A-a. To get 2A-b we only have to add the piece of information regarding catalexis. The right edge of the line would appear to be the unmarked locus for catalexis, so we assume that this fact also follows from the grammar. We can now rather easily specify the whole set of me-

\footnotetext{
${ }^{38}$ We have chosen an E meter here, since it will not end in a lapsing verse foot. This makes 2A-b more unmarked than the O meter 6A-b, which is necessarily final catalectic, due to NOLAPSE: 6A-b: LL.H L.H.L LL.H L.H. $\varnothing$.
} 
ters in the large cluster 2, see (30). A sketch of how this works is given in (59). Meters having the verse foot L.LL.L instead of L.H.L will be specified as lexically violating NOLAPSE- $\sigma$. The frequent violation of NOLAPSE- $\mu$ is simply the grammatical compliance with the privileged NOCLASH. The inhibition of acatalectic amphibrachs at the end of stanzas is a grammatical wellformedness effect of NOLAPSE on the metrical template. To save space, we register distinctive violation with exclamation marks turned upside down ( $i$ '), one for each distinctive violation.

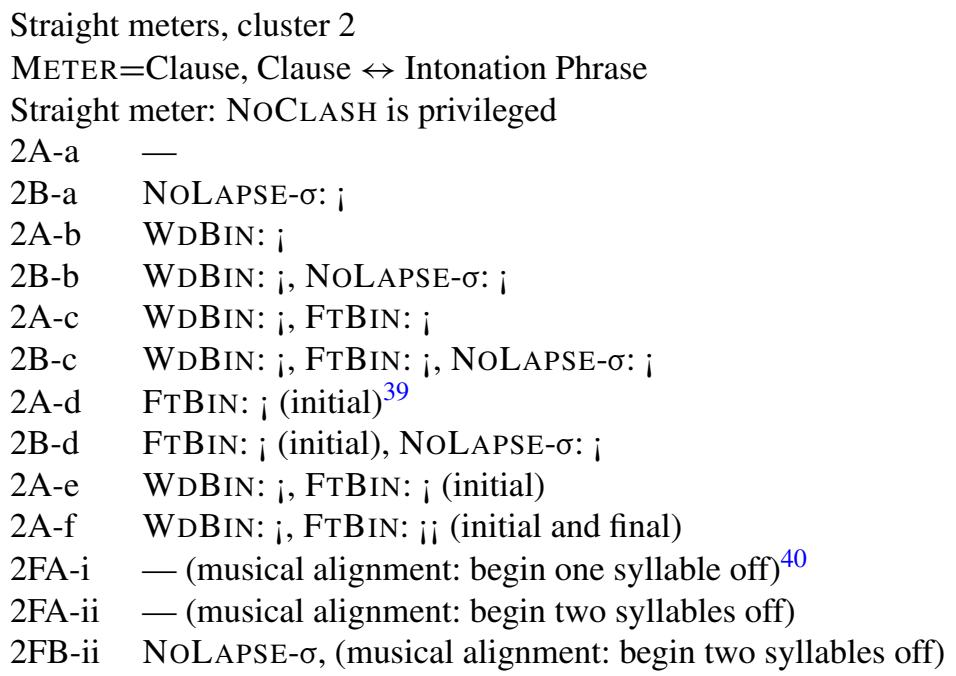

Notice that this understanding of meter considers catalexis as a singular mechanismdistinctive violation of a binarity constraint - which may regulate line length at any prosodic level. ${ }^{41}$ Thus, independent meters that are shorter than 4 verse feet will be specified as having another distinctive binarity violation on PRPHBIN. Dependent meters, most of which consist of two verse feet could either be viewed as a different basic unit $(\mathrm{METER}=\mathrm{XP})$, or as carrying distinctive binarity violations on IPHBIN. This issue must be left as a loose end here, pending more research on the other meters of Tashlhiyt, and their relation to the music. The solution to this issue could also lead us to a proposal for the few meters that are longer than four verse feet, e.g. whether they are made from a larger unit or perhaps a combination of meters. For a general discussion of markedness expressed as distinctive violation, see Golston (1996), and for meter in particular, see Golston and Riad (2000, 2005).

\footnotetext{
${ }^{39}$ Initial catalexis is more marked than final catalexis. I leave specification of how this works to the side here and only register 'initial' where relevant.

${ }^{40}$ Preliminarily, this property is attributed to the alignment of meter to music, cf. Sect. 6.3. More study is required, but at this point it seems unlikely the crucially fitted count should be derived from grammar, rather than from meter/text to music alignment.

${ }^{41}$ The traditional notion of catalexis refers only to syllables or moras.
} 


\section{Conclusion}

The results of our analysis of the straight meters of Tashlhiyt Berber can now be summarized. We have found that these meters are binary to a great extent, and we have argued that this fact derives from grammar in the same way that tendencies toward unmarkedness emerge in many of the more familiar prosodic morphemes. The markedness that distinguishes the individual meters mostly concerns line length, and we have expressed this property as the distinctive violation of binarity constraints, whenever it deviates from the unmarked. We can thus generalize over the traditional notions of catalexis and line length, via the notion of distinctive binarity violation, applying on different binarity constraints (see Golston and Riad 2000). If this generalization holds up, it makes for a simpler theory of line length than that offered by models that separate catalexis from line length, and which generate line length by another mechanism than grammar (Hanson and Kiparsky 1996; Fabb and Halle 2008; Blumenfeld 2015). Line length via unmarked binarity and distinctive violation makes the prediction that binary meters should be preferable, as a matter of linguistic markedness. This grounding of meter in the grammar also makes predictions regarding the preferable length of meters, namely as close to the preferred or average length of some prosodic category of the language.

The influence of the individual language on the meter is always acknowledged, but our approach ties meter more tightly to the grammar than previous approaches. This shows up in two respects. Firstly, the metrical template as such is a product of the grammar, in that it is a clause-size prosodic morpheme, which is realized simultaneously with regular prosody. Lexical, marked properties can always be added, of course, just as they can in other familiar cases of prosodic morphology. Secondly, grammatical constraints can be singled out as privileged for a given meter. A constraint of the grammar is thus made mandatory directly in the metrical structure. This leads to an overall prosodic improvement in verse compared to prose, on the particular constraint selected. The strong prediction here is that the guiding principle of meters, at least those made via improvement, should always be found within the grammar of the language. This prediction is not made without further stipulation by theories that employ matching between (properties of) prosodic structure and a (nonlinguistic) metrical template.

Turning to the straight meters of Tashlhiyt Berber, we have argued that the privileged constraint for this class is NoCLASH. This result stands as an empirical improvement irrespective of its theoretical interpretation. The role of NoCLASH grounds the previous generalizations (NoHH, ALTERN, and part of L4m) in a basic rhythmic principle that makes sense of the central aspects of the straight meters. We have also found grammatical effects of NOLAPSE in the analysis of these meters: preference for L.H.L over L.LL.L, and strict avoidance of stanza-final acatalectic lapsing verse feet, where the privileged NOCLASH has no say.

The understanding of this set of meters is also deepened by recognizing the fact that they circumscribe a coherent metrical space, consisting of minimally different surface meters. We have proposed that they are all derived from a single abstract meter. This is anticipated in Dell and Elmedlaoui (2008), who both recognize the straight meters as a natural class, and who meticulously list the variations found in 
their corpus. The notion of an abstract meter in this respect comes from Deo (2007) and her study of Sanskrit meters. We have suggested that the very notion of line length is a variable in the Tashlhiyt straight meters, unlike the Sanskrit meters. If this turns out to be true of other systems as well, then it calls into question the central status given to line length in some models of metrics (e.g. Fabb and Halle 2008).

Acknowledgements Much of the research for this article was carried out during my stay at Université Paris 8, Saint-Denis, in the spring of 2011. I would like to thank Jean-Louis Aroui and François Dell for stimulating discussion of Tashlhiyt Berber and metrics in general. François Dell read the article in manuscript form and provided invaluable help with numerous suggestions for improvement as well as several corrections. I have also profited from the expertise of Mohamed Elmedlaoui on several issues. Thanks to Chris Golston, Paul Kiparsky and Lev Blumenfeld for discussion and comments that have greatly influenced my analysis. I would also like to gratefully acknowledge the very useful guidance of the editor and three anonymous reviewers for Natural Language and Linguistic Theory. I am responsible for all remaining mistakes.

Open Access This article is distributed under the terms of the Creative Commons Attribution 4.0 International License (http://creativecommons.org/licenses/by/4.0/), which permits unrestricted use, distribution, and reproduction in any medium, provided you give appropriate credit to the original author(s) and the source, provide a link to the Creative Commons license, and indicate if changes were made.

\section{References}

Bennett, Ryan, Emily Elfner, and Jim McCloskey. 2016. Lightest to the right: Apparently anomalous displacement in Irish. Linguistic Inquiry 47(2): 169-234.

Bensoukas, Karim. 2001. Stem forms in the nontemplatic morphology of Berber. Diss., Mohammed V University, Rabat.

Blumenfeld, Lev. 2015. Meter as faithfulness. Natural Language and Linguistic Theory 33: 79-125.

Daunt, Marjorie. 1946. Old English verse and English speech rhythm. Transactions of the Philological Society 1946: 56-72.

Dell, François, and Mohamed Elmedlaoui. 1992/2001. Quantitative transfer in the nonconcatenative morphology of Imdlawn Tashlhiyt Berber. Journal of Afroasiatic Languages 3: 89-125. Reprinted 2001 in Phonology: Critical concepts, vol. 3, ed. Charles Kreidler, 535-579. Routledge.

Dell, François, and Mohamed Elmedlaoui. 2002. Syllables in Tashlhiyt Berber and in Moroccan Arabic. Vol. 2 of Kluwer international handbooks in linguistics. Dordrecht: Kluwer Academic.

Dell, François, and Mohamed Elmedlaoui. 2008. Poetic meter and musical form in Tashlhiyt Berber songs. Köln: Rüdiger Köppe.

Dell, François, and Mohamed Elmedlaoui. 2013. Syllables and gemination in imperfective stems in Tashlhiyt Berber. Brill's Annual of Afroasiatic Languages and Linguistics 5: 1-29.

Deo, Ashwini S. 2007. The metrical organization of classical Sanskrit verse. Journal of Linguistics 43(1): 63-114.

Downing, Laura. 2006. Canonical forms in prosodic morphology. Vol. 12 of Oxford studies in theoretical linguistics. Oxford: Oxford University Press.

Downing, Laura, and Marten Mous. 2011. Challenges of Cushitic reduplication for generalized template theory. Brill's Annual of Afroasiatic Languages and Linguistics 3(1): 82-110.

Fabb, Nigel. 2002. Language and literary structure: The linguistic analysis of form in verse and narrative. Cambridge: Cambridge University Press.

Fabb, Nigel, and Morris Halle. 2008. Meter in poetry: A new theory. Cambridge: Cambridge University Press.

Flack, Kathryn. 2007. Templatic morphology and indexed markedness constraints. Linguistic Inquiry 38: 749-758.

Fleisch, Henri S. J. 1956/1968. L'Arabe classique: Esquisse d'une structure linguistique. Beyrouth: Imprimerie Catholique. Reprinted 1968. Beyrouth: Dar El-Machareq Editerus.

Frota, Sónia, and Marina Vigário. 2007. Intonational phrasing in two varieties of European Portuguese. In Tones and tunes, vol. I, eds. Tomas Riad and Carlos Gussenhoven, 265-291. Berlin: de Gruyter. 
Ghomeshi, Jila, Ray Jackendoff, Nicole Rosen, and Kevin Russell. 2004. Contrastive focus reduplication in English (the salad-salad paper). Natural Language and Linguistic Theory 22: 307-357.

Golston, Chris. 1996. Direct optimality theory: Representation as pure markedness. Language 72(4): 713748.

Golston, Chris, and Tomas Riad. 1997. The phonology of classical Arabic meter. Linguistics 35: 111-132.

Golston, Chris, and Tomas Riad. 2000. The phonology of classical Greek meter. Linguistics 38(1): 99167.

Golston, Chris, and Tomas Riad. 2005. The phonology of Greek lyric meter. Journal of Linguistics 41: 77-115.

Golston, Chris, and Tomas Riad. 2016, Binary and unary structure in Classical Arabic metrics. Presentation given at the Old World Conference in Phonology (OCP) 13, Budapest.

Gordon, Matthew, and Latifa Nafi. 2012. Acoustic correlates of stress and pitch accent in Tashlhiyt Berber. Journal of Phonetics 40: 706-724.

Grice, Martine, Rachid Ridouane, and Timo B. Roettger. 2015. Tonal association in Tashlhiyt Berber: Evidence from polar questions and contrastive statements. Phonology 32(2): 241-266.

Halporn, James W., Martin Ostwald, and Thomas G. Rosenmeyer. 1980. The meters of Greek and Latin poetry, revised edn. Norman: University of Oklahoma Press.

Hanson, Kristin, and Paul Kiparsky. 1996. A parametric theory of poetic meter. Language 72(2): 287-335.

Hayes, Bruce. 1987. A revised parametric metrical theory. In North East Linguistic Society (NELS) eds. J. McDonough and B. Plunkett. Vol. 17.1 of NELS, 274-289.

Hayes, Bruce. 1995. Metrical stress theory: Principles and case studies. Chicago: The University of Chicago Press.

Hayes, Bruce, and Abigail Kaun. 1996. The role of phonological phrasing in sung and chanted verse. The Linguistic Review 13: 243-303.

Hayes, Bruce, and Margaret MacEachern. 1998. Quatrain form in English folk verse. Language 74(3): 473-507.

Itô, Junko, and Armin Mester. 2006. Prosodic adjunction in Japanese compounds. In Formal approaches to Japanese linguistics, Vol. 4 (FAJL), Osaka.

Jebbour, Abdelkrim. 1999. Syllable weight and syllable nuclei in Tachelhit Berber of Tiznit. Cahiers de Grammaire 24. Phonologie: Théorie et variation 95-116.

Jouad, Hassan. 1993. La mélodie de l'attaque syllabique et l'intonation. In À la croisée des études libycoberbères, mélanges offert à P. Galand-Pernet et L. Galand, eds. Jeannine Drouin and Arleth Roth, 429-436. Paris: Geuthner.

Jouad, Hassan. 1995. Le calcul inconscient de l'improvisation Poésie berbère-rythme, nombre et sens. Paris: Peeters.

Kager, René. 1993. Alternatives to the iambic-trochaic law. Natural Language and Linguistic Theory 11: $381-432$.

Kiparsky, Paul. 1977. The rhythmic structure of English verse. Linguistic Inquiry 8(2): 189-247.

Kiparsky, Paul. 2006. A modular metrics for folk verse. In Formal approaches to poetry, eds. Elan Dresher and Nila Friedberg, 7-49. Berlin: de Gruyter.

McCarthy, John, and Alan Prince. 1986. Prosodic morphology. Ms., University of Massachusetts, Amherst, and Brandeis University.

McCarthy, John, and Alan Prince. 1990. Foot and word in prosodic morphology: The Arabic broken plural. Natural Language and Linguistic Theory 8: 209-283.

McCarthy, John, and Alan Prince. 1994. The emergence of the unmarked: Optimality in prosodic morphology. Ms., University of Massachusetts, Amherst and Rutgers University.

Myrberg, Sara, and Tomas Riad. 2015. The prosodic hierarchy of Swedish. Nordic Journal of Linguistics 38(2): 115-147.

Piera, Carlos. 2001. Intonational factors in metrics. Belgian Journal of Linguistics 15(1): 205-228.

Prieto, Pilar. 2006. Phonological phrasing in Spanish. In Optimality-theoretic advances in Spanish phonology, eds. Sonia Colina and Fernando Martínez-Gil, 39-60. Amsterdam: John Benjamins.

Prince, Alan. 1983. Relating to the grid. Linguistic Inquiry 14(1): 19-100.

Prince, Alan, and Paul Smolensky. 1993. Optimality theory: Constraint interaction in generative grammar. Ms., Rutgers University and University of Colorado, Boulder.

Raven, David S. 1962. Greek metre. London: Faber \& Faber.

Riad, Tomas. 2013. Sköna och osköna ljud i skönlitteraturen. In Det sköna med skönlitteraturen, ed. Lars Rydquist. Vol. 3. Stockholm: Norstedts.

Selkirk, Elizabeth. 2000. The interaction of constraints on prosodic phrasing. In Prosody, theory and experiment: Studies presented to Gösta Bruce, ed. Merle Home, 231-261. Dordrecht: Kluwer Academic. 
Selkirk, Elisabeth, and Gorka Elordieta. 2010. The role for prosodic markedness constraints in phonological phrase formation in two pitch accent languages. Handout from presentation given at TIE 4 (The 4th European Conference on Tone and Intonation), Stockholm.

Swift, Nicholas. 2013. Responsion. Encyclopedia of ancient Greek language and linguistics. Leiden: Brill. Thompson, John. 1961. The founding of English metre. London: Routledge. 